\title{
Vertical Handoff between 802.11 and 802.16 Wireless Access Networks
}

\author{
by \\ Yongqiang Zhang
}

\author{
A thesis \\ presented to the University of Waterloo \\ in fulfillment of the \\ thesis requirement for the degree of \\ Master of Applied Science \\ in \\ Electrical and Computer Engineering
}

Waterloo, Ontario, Canada, 2008

(c) Yongqiang Zhang 2008 
I hereby declare that I am the sole author of this thesis. This is a true copy of the thesis, including any required final revisions, as accepted by my examiners.

I understand that my thesis may be made electronically available to the public. 


\begin{abstract}
Heterogeneous wireless networks will be dominant in the next-generation wireless networks with the integration of various wireless access networks. Wireless mesh networks will become to a key technology as an economically viable solution for wide deployment of high speed, scalable and ubiquitous wireless Internet services. In this thesis, we consider an interworking architecture of wireless mesh backbone and propose an effective vertical handoff scheme between 802.11 and 802.16 wireless access networks. The proposed vertical handoff scheme aims at reducing handoff signaling overhead on the wireless backbone and providing a low handoff delay to mobile nodes. The handoff signaling procedure in different scenarios is discussed. Together with call admission control, the vertical handoff scheme directs a new call request in the 802.11 network to the 802.16 network, if the admission of the new call in the 802.11 network can degrade quality-of-service (QoS) of the existing realtime traffic flows. Simulation results demonstrate the performance of the handoff scheme with respect to signaling cost, handoff delay, and QoS support.
\end{abstract}




\section{Acknowledgements}

First and foremost, I would like to express my deep gratitude and appreciation to my supervisor, Professor Weihua Zhuang, for her continuous guidance, encouragement, and patience, and for giving me the opportunity to do this work. Without

her advice and support not only in academic matters but also in personal matters, I am sure I would not be able to come to this point finishing my thesis.

I would also like to thank Professor Liang-Liang Xie and Professor Xuemin (Sherman) Shen for reviewing this thesis.

It is my great honor to be a member of Broadband and Communication Research (BBCR) group at the University of Waterloo. I would like to thank my friends for their friendship, help and discussions, especially Wei Song, Ping Wang, Atef Abdrabou, and Md. Forkan Uddin. Thanks also go to the administrative support staffs, Wendy Boles and Karen Schooley, for their attention and assistance.

I would like to acknowledge the research grant from the Bell University Labs (BUL) at the University of Waterloo, which supported this research.

Finally, my deepest gratitude and love belong to my wife and my parents for their support, encouragement, and endless love. 
To my truly loved wife and parents,

for their support, encouragement, and endless love. 


\section{Contents}

1 Introduction 1

1.1 Research Motivation . . . . . . . . . . . . . . 2

1.2 Thesis Outline . . . . . . . . . . . . . . . 4

2 Background 5

2.1 Wireless Mesh Network . . . . . . . . . . . . . . . . 5

2.2 Interworking . . . . . . . . . . . . . . . . 6

2.3 Mobility Management . . . . . . . . . . . . . . . . . . . . 9

2.4 Handoff . . . . . . . . . . . . . . . . . . . . . . . . 13

3 System Model and Problem Statement 16

3.1 System Architecture . . . . . . . . . . . . . . . . . 16

3.2 Mobility Scenario . . . . . . . . . . . . . . . . . 18

3.3 QoS Support ......................... 21

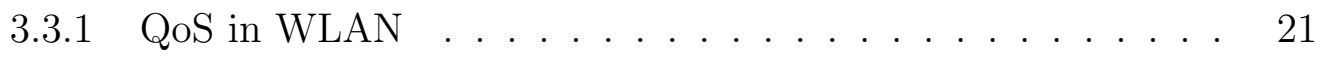

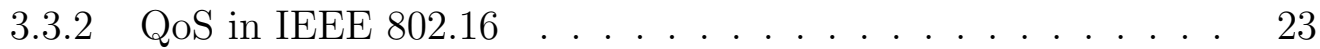

3.4 Problem Statement . . . . . . . . . . . . . . . 25 
4.1 Handoff Procedure . . . . . . . . . . . . . . . . . . . . . 28

4.1.1 New network interface scanning . . . . . . . . . . . . . 29

4.1 .2 New access router discovery $\ldots \ldots \ldots$

4.1 .3 New network entry . . . . . . . . . . . . . . . . 31

4.1.4 Updating routing information . . . . . . . . . . . . . 32

4.1.5 Example of handoff signaling procedure . . . . . . . . . . 35

4.2 Handoff Decision . . . . . . . . . . . . . . . . . . . . . . . 39

4.2.1 Vertical handoff for a new call . . . . . . . . . . . . . 47

4.2.2 Vertical handoff decision algorithm . . . . . . . . . 50

4.3 Summary . . . . . . . . . . . . . . . . . . . . 53

5 Perfomance Evaluation $\quad 55$

5.1 Simulation Configuration . . . . . . . . . . . . . . 55

5.2 Simulation Results . . . . . . . . . . . . . . . . . 58

6 Conclusions and Future Work $\quad 67$

6.1 Conclusions . . . . . . . . . . . . . . . . . . . 67

6.2 Future Work . . . . . . . . . . . . . . . . . . . . . 68

$\begin{array}{ll}\text { References } & 69\end{array}$ 


\section{List of Tables}

3.1 Access Category . . . . . . . . . . . . . . . . . . . 22

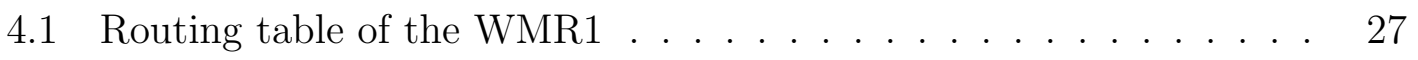

4.2 Information list of the neighboring edge WMR . . . . . . 28

4.3 The routing table before and after the vertical handoff in case $2 \ldots 38$

4.4 The routing table in the old network in case $3 \ldots \ldots \ldots$

4.5 The routing table in the new network in case $3 \ldots \ldots \ldots 42$

5.1 IEEE 802.11 e simulation parameters $\ldots \ldots \ldots \ldots \ldots$

5.2 Signaling cost and delay in Layer 3 handoff $\ldots \ldots \ldots \ldots$. . . . . 64 


\section{List of Figures}

3.1 The system architecture of wireless mesh backbone. . . . . . . . . . 17

3.2 The functions of the WMR and its protocol stack. . . . . . . . . 18

3.3 The macro-mobility and micro-mobility scenario. . . . . . . . . . 19

4.1 Route change and signal control in vertical handoff with the same WMR. . . . . . . . . . . . . . . . 36

4.2 Vertical handoff between Intra Mesh Routers. . . . . . . . . . . . . 37

4.3 Signaling for vertical handoff from WLAN to WiMAX between Intra Mesh Routers. . . . . . . . . . . . . . . . . . . 39

4.4 Vertical handoff between Inter Mesh Routers. . . . . . . . . . . . . 40

4.5 Signaling for vertical handoff from WLAN to WiMAX between Inter Mesh Routers. . . . . . . . . . . . . . . . . . . . 41

4.6 Joint admission control and vertical handoff procedure. . . . . . . . 47

4.7 Admission control decision algorithm in the QAP. . . . . . . . . 49

4.8 Admission control decision algorithm in BS. . . . . . . . . . . 51

4.9 Vertical handoff decision algorithm from WLAN to WiMAX. . . . . 52

4.10 Vertical handoff decision algorithm from WiMAX to WLAN. . . . . 53

5.1 The dual-interface MN model in the simulation. . . . . . . . . . 56

5.2 The maximum number of $\mathrm{MN}$ in 802.11 with QoS support. . . . . . 59 
5.3 The system throughput versus traffic load. . . . . . . . . . . . 60

5.4 The packet delay of the first MN during the vertical handoff jointly with admission control. . . . . . . . . . . . . . . . . . . 61 61

5.5 The RSS from AP When the first MN is moving out/in the doublecoverage area. . . . . . . . . . . . . . . . . . . 62

5.6 The packet delay of the first MN When it is moving out/in the double-coverage area. . . . . . . . . . . . . . . . . 63

5.7 The system throughput When the first MN is moving out/in the double-coverage area. . . . . . . . . . . . . . . . . . 63 


\section{List of Abbreviations}

$\begin{array}{ll}\text { AC } & \text { Access categorie } \\ \text { ACK } & \text { Acknowledgement } \\ \text { ACM } & \text { Admission control mandatory } \\ \text { ADDTS } & \text { Add traffic stream } \\ \text { AIFS } & \text { Arbitration interframe space } \\ \text { AP } & \text { Access point } \\ \text { BE } & \text { Best effort } \\ \text { BS } & \text { Base station } \\ \text { CAC } & \text { Call admission control } \\ \text { CAR } & \text { Candidate access router } \\ \text { CARD } & \text { Candidate access router discovery } \\ \text { CAP } & \text { Controlled access phases } \\ \text { CBR } & \text { Constant bit rate } \\ \text { CDMA } & \text { Code-division multiple access } \\ \text { CDF } & \text { Cumulative distribution function } \\ \text { CFP } & \text { Contention-free period } \\ \text { CID } & \text { Connection identifier } \\ \text { CN } & \text { Correspondent node } \\ \text { CoA } & \text { Care-of address } \\ \text { CP } & \text { Contention period } \\ \text { CSMA } & \text { Carrier sense multiple access } \\ \end{array}$




\begin{tabular}{|c|c|}
\hline CSMA/CA & CSMA with collision avoidance \\
\hline CTS & Clear to send \\
\hline CW & Contention window \\
\hline DCD & Downlink channel descriptor \\
\hline DCF & Distributed coordination function \\
\hline DHCP & Dynamic host configuration protocol \\
\hline DIFS & Distributed interframe space \\
\hline DL-MAP & Downlink map message \\
\hline DSL & Digital subscriber line \\
\hline EDCA & Enhanced distributed channel access \\
\hline EDCAF & Enhanced distributed channel access function \\
\hline FA & Foreign agent \\
\hline FBU & Fast binding update \\
\hline FDD & Frequency-division duplex \\
\hline GPRS & General packet radio service \\
\hline HA & Home agent \\
\hline HAck & Handover acknowledge \\
\hline HCCA & HCF controlled channel access \\
\hline HCF & Hybrid coordination function \\
\hline HI & Handover initiate \\
\hline HMIPv6 & Hierarchical mobile IPv6 mobility management \\
\hline IETF & Internet engineering task force \\
\hline IDMP & Intra-domain mobility management protocol \\
\hline IP & Internet protocol \\
\hline L2 & Link layer, Layer 2 \\
\hline L3 & IP layer, Layer 3 \\
\hline LCoA & On-link care-of address \\
\hline MAC & Medium access control \\
\hline
\end{tabular}




$\begin{array}{ll}\text { MAP } & \text { Mobility Anchor Point } \\ \text { MGW } & \text { Mesh gateway } \\ \text { MIHF } & \text { Media independent handoff function } \\ \text { MMN } & \text { Wireless mesh network } \\ \text { MN } & \text { Mobile node } \\ \text { MSDU } & \text { MAC Service Data Unit } \\ \text { NIC } & \text { Network interface card } \\ \text { NNPI } & \text { New network prefix information } \\ \text { nrtPS } & \text { Non-real-time polling service } \\ \text { NWMR } & \text { New wireless mesh router } \\ \text { OSI } & \text { Open systems interconnection } \\ \text { PCF } & \text { Point coordination function } \\ \text { PDF } & \text { Probability density function } \\ \text { PDU } & \text { Protocol data unit } \\ \text { PMP } & \text { Point-to-multipoint } \\ \text { PrRtAdv } & \text { Proxy Router Advertisement message } \\ \text { PWMR } & \text { Previous wireless mesh router } \\ \text { QAP } & \text { QoS access point } \\ \text { QBSS } & \text { QoS basic service set } \\ \text { QoS } & \text { Quality of service } \\ \text { QSTA } & \text { QoS station } \\ \text { RCoA } & \text { Regional care-of address } \\ \text { RSS } & \text { Received signal strength } \\ \text { RTP } & \text { Real-time transport protocol } \\ \text { rtPS } & \text { Real-time polling service } \\ \text { RTS } & \text { Request to send } \\ \text { RtSoIPr } & \text { Router Solicitation for Proxy Advertisement message } \\ \text { SIFS } & \text { Short interframe space } \\ & \\ \text { MW } & \end{array}$




\begin{tabular}{|c|c|}
\hline SIP & Session initiation protocol \\
\hline SS & Subscriber station \\
\hline TCP & Transmission control protocol \\
\hline TDD & Time-division duplex \\
\hline TDMA & Time-division multiple access \\
\hline TS & Traffic stream \\
\hline TSPEC & Traffic specification \\
\hline TXOP & Transmission opportunity \\
\hline UCD & Uplink channel descriptor \\
\hline UDP & User datagram protocol \\
\hline UGS & Unsolicited grant service \\
\hline UL-MAP & Uplink map message \\
\hline UMTS & Universal mobile telecommunications system \\
\hline URI & Updating routing information \\
\hline VolP & Voice over IP \\
\hline Wi-Fi & Wireless-fidelity \\
\hline WiMAX & Worldwide interoperability for microwave access \\
\hline WLAN & Wireless local area network \\
\hline WMAN & Wireless metropolitan area network \\
\hline WMR & Wireless mesh router \\
\hline
\end{tabular}




\title{
List of Symbols
}

\author{
$A C K \quad$ The packet size of ACK message in 802.11e \\ AIFS Arbitration Interframe Space Period in 802.11e \\ $B_{M} \quad$ A portion of $B_{U}$ \\ $B_{N R} \quad$ Normalized bandwidth requirement of the new real-time traffic \\ $B_{U} \quad$ The maximal channel utilization \\ $B_{u s} \quad$ The total bandwidth which has been used by the admitted real-time \\ traffic \\ $C_{n r t P S} \quad$ Total capacity (bps) allocated for current nrtPS connections \\ $C_{r t P S} \quad$ Total capacity (bps) allocated for current rtPS connections \\ $C_{U G S} \quad$ Total capacity (bps) allocated for current UGS connections \\ $C_{\text {uplink }} \quad$ Total capacity (bps) allocated for uplink transmission \\ $G_{r} \quad$ The antenna gain of the receiver \\ $G_{t} \quad$ The antenna gain of the transmitter \\ $H \quad$ The size of MAC header in 802.11e \\ $h_{r} \quad$ The heights of the receive antenna \\ $h_{t} \quad$ The heights of the transmit antenna \\ $L \quad$ The system loss \\ $r_{i} \quad$ Average data rate of new service flow or connection request \\ $R_{P b} \quad$ The basic rate in $802.11 \mathrm{e}$ \\ $R_{P h} \quad$ The PHY data rate in 802.11e \\ $S B A \quad$ Surplus Bandwidth Allowance
}


SIFS Short Interframe Space Period in 802.11e

$T_{802.11} \quad$ The one-way average packet delay on the 802.11 link

$T_{802.16} \quad$ The one-way average packet delay on the 802.16 link

$T_{H A-F A} \quad$ The message transmitting time for updating the route from HA to FA

$T_{H A-W M R 3}$ The message transmitting time for updating the route from HA to WMR3

$T_{\text {link }} \quad$ The one-way link delay between the wired nodes in simulation

$T_{M N-H A} \quad$ The message transmitting time for updating the route from MN to HA

$T_{M N-F A} \quad$ The message transmitting time for updating the route from MN to FA

$\begin{array}{ll}P_{r} & \text { The received power in the MN }\end{array}$

$P_{t} \quad$ The transmitted signal power 


\section{Chapter 1}

\section{Introduction}

Next-generation wireless networks have been envisioned as an Internet Protocol (IP) based infrastructure with the integration of various wireless access networks such as IEEE 802.11 wireless local area networks (WLANs), IEEE 802.16 wireless metropolitan area networks (WMANs), General Packet Radio Service (GPRS), and Universal Mobile Telecommunications System (UMTS). Heterogeneous wireless networks need to cooperate to provide users with seamless mobility and required quality of service (QoS). Mobile nodes (MNs) can automatically switch the connectivity between different types of networks. Such future mobile communications calls for reconfigurable and efficient systems. It is expected that wireless mesh networks (WMNs) will become a key technology as an economically viable solution for wide deployment of high speed, scalable, and ubiquitous wireless Internet services.

Mesh routers and mesh clients are the two types of nodes in WMNs. A mesh router not only has gateway/bridge functions, but also has routing functions to support mesh networking. It is usually equipped with multiple wireless interfaces built on either the same or different wireless access technologies to improve the flexibility of mesh networking. Mesh routers have minimal mobility, form a multihop network topology, and can self-configure a wireless broadband mesh backbone for local communication and information delivery to/from a wired Internet backbone via wired gateways. The wireless backbone provides multi-hop connectivity 
between a mobile client and a gateway.

Moreover, the integration of WMNs with various existing wireless networks such as cellular, wireless-fidelity (Wi-Fi), and worldwide inter-operability for microwave access (WiMAX) networks can be accomplished through the gateway/bridge functionalities in mesh routers. With the integration of multiple wireless access technologies, the wireless mesh backbone provides a viable solution for users to access the Internet anywhere anytime. Compared to wired networks, e.g., cable or optical networks, the wireless mesh backbone is an economic alternative to broadband networking, especially in underdeveloped regions. Existing WLANs can provide low-cost data services and have been widely deployed in traffic hotspots such as offices, hotels, shopping malls, schools, university campus, and airports. On the other hand, IEEE 802.16 can provide high speed wireless services in wide areas. As a result, the integration between a wireless mesh backbone (based on IEEE 802.16 standards) and WLANs can create a complete wireless solution for delivering broadband Internet services to the hotspots instead of cable, DSL, and T1 level services.

\subsection{Research Motivation}

The interworking between different wireless access networks has been a hot research and development topic in the past few years. Different radio access technologies present distinct characteristics in terms of mobility management, security support, and QoS provioning. To achieve seamless mobility and end-to-end QoS guarantee for the users, these issues should be carefully addressed while developing the interworking and handoff schemes of WMNs with various wireless networks. Mesh routers in the WMNs play an important role. The 802.11 access point (AP) functions and 802.16 base station (BS) functions can be integrated into one mesh router. When an MN switches the network interface, only the link type is changed between the MN and mesh routers, and the MN still connects to the same mesh router. In 
this case, the traditional mobility management such as Mobile IP leads to a large handoff delay with too much signaling cost. Thus to achieve fast and seamless handoff, a new handoff scheme should be considered.

Another factor which can affect seamless vertical handoff is how and when to make a handoff decision. In traditional handoff, the received signal strength is the main handoff metric. However, in vertical handoff, only the received signal strength is not enough to make a handoff decision. The handoff metrics may be cost of service, available bandwidth, power requirements, QoS and user preference. It is a challenge to develop a vertical handoff decision algorithm for optimal radio resource utilization with various QoS support. The vertical handoff may not take place only at the cell edge. It can occur at any time (even when the MN does not move) depending on the network condition and user preference such as in a situation of network congestion. How to make a decision to trigger a vertical handoff according to the system performance and QoS parameters becomes the main part of this kind of vertical handoff. Therefore an effective and efficient vertical handoff decision algorithm in the interworking between 802.11 and 802.16 in WMN is needed to maximize the resource utilization.

In this thesis, we present an interworking architecture of wireless mesh backbone and propose an effective vertical handoff scheme between 802.11 and 802.16. The proposed vertical handoff scheme aims at reducing handoff signaling overhead on the wireless backbone and providing a low handoff delay to MNs.

Admission control is a QoS mechanism that protects the QoS of existing traffic flows and decides whether a new call can be admitted. In this thesis, we use admission control results to make the handoff decision for the $\mathrm{MN}$ in the overlay area of the two networks when the MN starts a new call. Our proposed handoff decision algorithm combined with admission control can switch new calls to WiMAX to guarantee QoS support to the existing traffic flows in WLAN. Therefore the QoS support can be provided to as many users as possible. 


\subsection{Thesis Outline}

The remaining of the thesis is organized as follows:

In Chapter 2, an overview of WMNs are breifly presented, followed by the interworking architecture of heterogeneous networks in the literature. The backgound of the mobility management and handoff in heterogeneous networks is also reviewed.

In Chapter 3, the system model of the wireless mesh backbone is proposed and the different mobility scenarios are presented. The QoS support of 802.11e and 802.16e link is introduced. The problem statement sumarizes the problems in vertical handoff in the proposed system model.

In Chapter 4, an effective vertical handoff scheme between 802.11 and 802.16 is proposed, which includes the handoff signaling procedure and handoff decision. The handoff signaling procedure in different scenarios is discussed in details, followed by the handoff decision algorithm combined with admission control.

In Chapter 5, the simulation is conducted to evaluate the performance of the proposed vertical handoff scheme. Simulation results demonstrate that the newly proposed vertical handoff scheme performs well with respect to signaling cost, handoff delay, system throughput, and packet delay.

In Chapter 6, the thesis is concluded, followed by the directions of further work. 


\section{Chapter 2}

\section{Background}

\subsection{Wireless Mesh Network}

A WMN is dynamically self-organized and self-configured, with the mesh routers and mesh clients automatically establishing and maintaining mesh connectivity among themselves. In a WMN, mesh routers form the wireless mesh backbone. Mesh clients operate as a host with necessary functions for mesh networking and also can work as routers forwarding packets on behalf of other nodes that may not be within direct wireless transmission range of their destinations. Such feature brings many advantages such as low up-front cost, easy network maintenance, robustness, and reliable service coverage.

WMNs are classified to three types in [1]: Infrastructure/Backbone, Client, and Hybrid WMNs. In Infrastructure/Backbone WMNs, mesh routers form a backbone network to clients. Mesh routers can use different radio technologies for backbone and user communication. The mesh backbone is meant to be self-configuring, selfhealing and to offer gateway functionality for connections to wired networks such as the Internet. In Client WMNs, all the nodes are counted as mesh clients which can perform routing and configuration functionalities. In fact, a Client WMN is a traditional ad hoc network without infrastructure. Hybrid WMNs combine the above two WMNs. The mesh client can route the traffic for the other clients which 
cannot connect to the mesh backbone directly. This increases the coverage and connectivity of the networks.

The wireless mesh backbone forming the core of a WMN provides a backhaul communication service to the Internet and multi-hop connectivity between a mobile client and a gateway. It is different from the conventional ad hoc networks due to node mobility. It covers a potentially much larger area than home, enterprise, building, or community networks. Also, in a wireless mesh backbone, the mesh routers usually do not have strict constraints on power consumption. Therefore, the MAC or routing protocols optimized for mesh routers may not be suitable for mesh clients.

The small coverage range of existing WLANs limits the mobility of the MNs. Increasing the number of the hotspots will increase the deployment costs due to the wired connections between the hotspots and Internet. IEEE 802.16 can provide high speed Internet access in a wide area. The wireless mesh backbone based on the IEEE 802.16 standard can provide high speed network connections for WLAN hotspots.

Furthermore, if the MN has multiple network interface cards (NIC) (for example, a portable device can have a Wi-Fi NIC and a WiMAX NIC at the same time), the mobility support of the MN can be improved with seamless connectivity when the MN moves out of the range of a WLAN. Even in the overlay area within the coverage of both WLAN and WiMAX, the MN can change the connection depending on the network conditions and its QoS requirements. In this thesis, we mainly focus on how the MN changes the network interface in the overlay area according to the current network traffic load condition and the user QoS requirements.

\subsection{Interworking}

Different radio access technologies present distinct characteristics in terms of mobility management, security support, and QoS provioning. To achieve seamless mobility and end-to-end QoS guarantee for the users, these issues should be care- 
fully addressed while developing interworking schemes. The interworking between different wireless access networks has been a hot research topic in recent years. Most of the researchers mainly focus on interworking between WLAN and 3G cellular networks such as UMTS and CDMA2000, which are the two major standards for 3G mobile/cellular networks specified by the 3rd generation partnership projects, i.e., $3 \mathrm{GPP}$ and $3 \mathrm{GPP} 2$. There are two main ways of integrating the WLAN and 3G cellular networks [2], defined as tightly-coupled interworking and loosely-coupled interworking.

In tightly-coupled interworking, the WLAN appears to the $3 \mathrm{G}$ wireless core network as one of the $3 \mathrm{G}$ wireless radio access network. The WLAN emulates functions that are natively available in the cellular radio access network. The corresponding $3 \mathrm{G}$ protocols such as mobility management and authentication need to be implemented in the WLAN network cards and the 802.11 gateway. The WLAN data traffic goes through the $3 \mathrm{G}$ core network before reaching the Internet. Thus the different networks share the same authentication, signaling, transport and billing infrastructures. However, there are three disadvantages of tightly-coupled interworking: (1) both the 802.11 and $3 \mathrm{G}$ cellular network must be owned by the same operator due to the exposed $3 \mathrm{G}$ core interfaces to the WLAN; (2) a large volume of traffic from the WLAN to the $3 \mathrm{G}$ core network will lead to capacity problem; (3) the WLAN device should be modified in hardware and software. On the other hand, in loosely-coupled interworking, the WLAN connects to the Internet directly. The data paths are completely separated between WLAN and 3G wireless networks. The authentication, billing and mobility management in the two domains can be implemented by different mechanism and protocols. Therefore, the loosely-coupled interworking has several advantages in terms of minimal requirements to modify current WLAN standards, the flexibility and independence of implementing individually different mechanisms with each network, widespread coverage with roaming agreements of different service providers. However the loosely-coupled interworking may have high handoff latency due to a relatively long path which the mobility signaling may traverse. The hybrid-coupled interworking which is the combination 
of the tightly-coupled and loosely-coupled interworking is proposed in [3]. In the hybrid-coupled interworking scheme, traffic paths are differentiated according to the type of the traffic. For the real-time traffic, a tightly-coupled network architecture is chosen, and for the non-real time and bulky traffic, a loosely-coupled network architecture is chosen. It may support quality of service of real-time traffic and service continuity during vertical handover for WLAN users. However the drawback of tightly-coupled and loosely-coupled interworking still exist and the implementation of this architecture is complicated.

In the architecture of interworking between wireless mesh backbone and WLAN, the data traffic from the WLAN to the Internet goes through the wireless mesh backbone. Hence, in some sense, we can say it is a tightly-coupled interworking. However it is different from the tightly-coupled architecture of integrating the WLAN and $3 \mathrm{G}$ cellular network. The mesh routers play an important role. According to its gateway and bridge functions, no additional gateway for the WLAN (e.g., the one in the interworking between WLAN and 3G cellular network) is needed. The mesh router also acts as an AP for WLAN. An integrated AP which integrates a WMAN Subscriber Station and a WLAN AP is introduced in [4]. With routing functions for mesh networks, this integrated AP can be used as a mesh router in the wireless mesh backbone. When the MN moves out of the coverage of the WLAN, it can switch for a connection to the WMAN Subscriber Station.

In this thesis, a mesh router is assumed to have 802.11e AP functions, 802.16e BS functions with PMP mode, routing functions, and 802.16e subscriber station (SS) functions with mesh mode. The MNs can connect only via mesh routers to access the Internet using two types of links: the IEEE 802.11e and the IEEE 802.16e links. The IEEE 802.16e links between MNs and mesh routers operate in the pointto-multipoint (PMP) mode, while the IEEE 802.16e links among neighboring mesh routers operate in the mesh mode. 


\subsection{Mobility Management}

Mobility scenarios can be classified into macro-mobility and micro-mobility. Macromobility is referred to as inter-domain mobility where an MN moves between different administrative domains. Micro-mobility is referred to as intra-domain mobility where an MN moves within one administrative domain.

Mobility management contains two components: location management and handoff management [5]. Location management enables the network to track the current attachment point of a mobile user. The first step is location registration (or location update). In this step, the mobile terminal periodically informs the network of its up-to-date location information, allowing the network to authenticate the user and update the users location profile. The second step is call delivery. The network determines the current location in which a mobile terminal is located so the incoming communication for the mobile terminal can be routed to the corresponding location. There are some challenges for the design of location management especially for inter-domain roaming in terms of the signaling overhead, call delivery latency, and QoS guarantees in different systems. Handoff management enables the network to maintain the on-going connection when a mobile terminal switches its access point. There are three stage processes for handoff. First the initiation for handoff is triggered by either the user, a network agent, or changing network conditions. The second stage is new connection generation, where the network must find new resources for the handoff connection and perform any additional routing operations. Finally data-flow control needs to maintain the delivery of the data from the old connection path to the new connection path according to agreed-upon service guarantees.

Mobility management in heterogeneous networks can take place in different layers of the OSI protocol stack reference model such as network layer, transport layer, and application layer. Several mobility protocols have been proposed for next-generation all-IP based wireless systems in the literature.

Mobile IP is a dominant network layer mobility protocol to solve the problems 
of receiving packets using the base IP protocol when an MN is moving. It has been a proposed standard for several years. It allows an MN to continue using its permanent home address as it moves around the Internet and supports transparency above the network layer. It introduces new functional entities: home agent (HA) and foreign agent (FA). Since the IP has been developed along two different ways: IPv4 and IPv6, the IETF working group in Mobile IP has also proposed two versions: Mobile IPv4 and Mobile IPv6. Due to the recent exponential growth of the Internet and the impending exhaustion of the IPv4 address space, it seems clear that the IPv6 and Mobile IPv6 will become dominant in the future.

In Mobile IPv4 [6], the HA is a router in the home network of an MN, which tunnels datagrams for delivery to the MN when it is away from home, and maintains current location information for the MN. The FA is a router in the visited network of the MN, which provides routing services to the MN while registered. When the MN detects that it has moved to a foreign network, it obtains a care-of address $(\mathrm{CoA})$ in the foreign network. The CoA can either be obtained from an FA's advertisement or by some external assignment mechanism such as Dynamic Host Configuration Protocol (DHCP). The MN then registers its new CoA with its HA through exchange of a Registration Request and Registration Reply message. Datagrams sent to the MNs home address are intercepted by its HA, tunneled by the HA to the MN. This is known as triangular routing problem. In the reverse direction, datagrams sent by the $\mathrm{MN}$ are generally delivered to their destination using standard IP routing mechanisms without passing through the HA.

In Mobile IPv6 [7], a binding update message from the MN is used to inform the HA and correspondent node $(\mathrm{CN})$ about the changes in the point of attachment. Both the HA and CN maintain their own binding cache. There are two ways in which MNs that are out of home can communicate with its CN in IPv6 networks: (1) The data can be sent directly between the $\mathrm{MN}$ and the $\mathrm{CN}$ since the $\mathrm{MN}$ registers its current binding at the $\mathrm{CN}$ if the $\mathrm{CN}$ is Mobile IPv6-capable. The direct method of data delivery is referred to as route optimization compared to the triangular 
routing via $\mathrm{HA}$ in Mobile $\mathrm{IPv}$. This method allows the shortest communications path to be used. It also eliminates congestion at the MN's HA and home link. In addition, the impact of any possible failure of the HA or networks is reduced; (2) If the $\mathrm{CN}$ is not Mobile IPv6-capable or the registration of the binding for the MN with the CN that is Mobile IPv6-capable has not yet been completed, then data can be sent indirectly between the $\mathrm{MN}$ and the $\mathrm{CN}$ via the HA. Data packets from the CN are routed to the HA and then tunneled to the MN. Data packets to the $\mathrm{CN}$ are tunneled from the MN to the HA ("reverse tunneled") and then routed normally from the home network to the $\mathrm{CN}$. This method is referred to as bidirectional tunneling.

However, there are some drawbacks in Mobile IP. The location update to the HA and CN may lead to a significant signaling cost when the number of MNs increases. The handoff latency may be very large if the foreign and home networks are far away from each other. Also, when the MN moves from one foreign network to another foreign network, the packets forwarded to the previous foreign link may be dropped since the previous foreign agent does not know the MN's movement. Due to the heavy signaling load and large handoff latency, Mobile IP is more suited for macro-mobility management but less well suited for micro-mobility management.

To support micro-mobility management in the environment where mobile hosts change their points of attachment to the network so frequently within one domain, IP micro-mobility protocols are designed to reduce the overhead which the Mobile IP introduces. To minimize poor performance during handoff, micro-mobility protocols complement Mobile IP by supporting local mobility. Many micro-mobility protocols have been proposed in the literature. The existing proposals for micromobility can be broadly classified into two types [8],[9]: routing-based and tunnelbased schemes. In routing-based schemes, packets are forwarded from the domain gateway to the MN by using host-specific routing within one domain. Cellular IP and HAWAII are two typical routing-based micro-mobility protocols. Tunnel-based schemes apply local or hierarchical registration and encapsulation concepts to re- 
duce the global signaling load and handoff latency. Mobile IP regional registration, hierarchical Mobile IP, and intra-domain mobility management protocol (IDMP) belong to the tunnel-based scheme.

Hierarchical Mobile IPv6 Mobility Management (HMIPv6) is the current IETF IPv6 micro-mobility proposal [10]. A new node called the Mobility Anchor Point (MAP), which is a router located in a network visited by an MN, is introduced as a new function. The MAP basically is used by an MN as a local HA. When an MN moves into a new MAP domain it needs to configure two CoAs: a regional care-of address (RCoA) on the MAP's link and an on-link care-of address (LCoA). The RCoA is the address that the $\mathrm{MN}$ will use to inform its $\mathrm{HA}$ and $\mathrm{CN}$ about its current location. The MAP will receive packets addressed to the MN's RCoA (from the $\mathrm{HA}$ or $\mathrm{CN}$ ) and tunnel them to the MN. When the MN performs a handoff between two access points within the same MAP domain, only the MAP has to be informed. There are some advantages in Hierarchical Mobile IPv6: (1) The signaling load is reduced when the MN changes the point of attachment in the same domain; (2) The handoff latency and packet loss may be reduced during the intra-domain handoff.

Transport layer and application layer mobility protocols are designed to provide mobility support independent of underlying network layer protocols. They offer an alternative for mobility management in the heterogeneous network. One transport layer mobility protocol is TCP-Migrate. Session initiation protocol (SIP) has been standardized by IETF as mobility support in an application layer. SIP is an endto-end orientated signaling protocol and can be applied for real time applications.

The above mobility protocols for different layers such as Mobile IP and SIP can be well applied in the loosely-coupled interworking architecture of WLAN and 3G integrated networks. HMIPv6 can improve the performance for micro-mobility. Performance of SIP in the WLAN-UMTS interworking has been studied in [11]. It shows that the handoff from WLAM to UMTS suffers much more delay than the handoff from UMTS to WLAN due to the transmission of SIP signaling messages 
over erroneous and bandwidth-limited wireless links. To support delay sensitive applications, soft handoff techniques need to be applied for SIP. In [12], a hybrid architecture has been proposed, incorporating both Mobile IP and SIP. This integration provides transparent mobility services for both real time and non real time applications.

In the interworking environment between wireless mesh backbone and WLAN in this thesis, there are several different mobility scenarios corresponding with macromobility and micro-mobility. To achieve fast and seamless handoff, a new vertical handoff scheme is considered by using some messages in the Mobile IP protocol.

\subsection{Handoff}

Handoff process can be seen as having two stages: (1) Handoff detection, and (2) Handoff execution. Handoff detection includes network discovery and handoff decision. Which kind of handoff metrics should be used and how to apply them to make the handoff decision are the main problems in handoff detection. In handoff execution, the mobility management plays an important role. The signaling over-

head and handoff latency are different in different mobility protocols. To achieve seamless and fast handoff, these two stages should be paid attention to. The time when the handoff decision is made can affect the overall performance of the handoff process such as packet loss. A heavy signaling overhead in handoff management leads to large handoff latency.

In heterogeneous networks, handoff takes place in two ways according to the radio access technologies. Horizontal handoff and vertical handoff have been defined in [13]. Horizontal handoff is between BSs or APs which use the same radio access technology, called intra-technology handoff. Vertical handoff is between BSs or APs which use different radio access technologies, called inter-technology handoff. The main difference between horizontal handoff and vertical handoff is symmetry. Because of the different characteristics of different access technologies, the vertical 
handoff is asymmetry.

In the interworking of heterogeneous networks, one of the major challenges is seamless vertical handoff. Several issues should be studied such as handoff metrics, handoff decision algorithms and handoff management in order to achieve seamless handoff. Vertical handoff is more complex than horizontal handoff. In traditional horizontal handoff, the main handoff metric is the received signal strength. However, in vertical handoff, only the received signal strength is not enough to make a handoff decision. The handoff metrics may include cost of service, available bandwidth, power requirements, quality of service, and user preference. The vertical handoff may not take place only at the cell edge. It can happen at any time, depending on the network condition and user preference. Such combined metrics lead to a challenge for designing a handoff decision algorithm because some factors are more difficult to obtain than the physical layer parameters such as received signal strength (RSS) and signal-to-interference ratio. In [14], a bandwidth measurement of WLAN and an QoS-based vertical handoff decision algorithm between WLAN and wide-area access network have been proposed by taking account of the network transport capability and user service requirements. In [15], the author uses a decision function which enable devices to assign weights to different network factors to make a handoff decision. It requires much empirical work. In [16], an adaptive multi-criteria handoff decision algorithm is designed by using fuzzy logic concept. In [17], a vertical handoff scheme between IEEE 802.16a networks and IEEE 802.11n networks is proposed. The available bandwidth in WLAN and the RSS are the metrics used for handoff decisions. It is assumed that the MN will stay in the IEEE $802.11 n$ networks as long as possible. So only the RSS is used for handoff from the IEEE 802.11n network to the IEEE 802.16a network.

After a handoff decision, the handoff management procedure is performed to change the network interface and maintain the on-going connection. The mobility management protocol is to implement this function. Some extensions or combinations of existing mobility protocols are proposed to implement vertical handoff in 
heterogeneous networks. In [18], an interworking architecture between WLAN and GPRS links is presented by introducing a simple extension to the exsiting Mobile IP implementation. A virtual network interface is used to tunnel packets to the foreign agent over a TCP connection because GPRS interface on an MN cannot be accessed directly by Mobile IP. The vertical handoff can take place by triggering a horizontal handoff between the WLAN interface and the virtual interface for GPRS. A SIP-based vertical handoff between WWAN and WLAN is performed with numerical analysis for handoff delay in [12]. In [17], vertical handoff is implemented by using the transport layer protocol SCTP (Stream Control Transmission Protocol) and the application layer protocol SIP.

In the interworking environment between a wireless mesh backbone and a WLAN, when the MN changes its network connection link between WLAN and WiMAX, the vertical handoff may be not based on the mobility protocols in the literature since the point of attachment is not changed. Moreover, how to make a decision to trigger a vertical handoff according to the QoS requirement is one of the main parts of this kind of vertical handoff. 


\section{Chapter 3}

\section{System Model and Problem}

\section{Statement}

\subsection{System Architecture}

The system model is illustrated in Figure 3.1. The wireless mesh routers (WMRs) form a wireless mesh backbone. The links between the WMRs are based on the IEEE 802.16e mesh mode standard. The WMR which is connected to the Internet with wired line is called Mesh Gateway (MGW). The WMRs can work as an AP for a WLAN based on the IEEE 802.11e standard to provide Wi-Fi service, which means that the AP for a WLAN is integrated in the WMR. The WMRs can also provide WiMAX services to the MNs. Therefore the MNs with the dual network interfaces can connect to the Internet through the WMRs by an IEEE 802.11e link or an IEEE 802.16e link. The WMRs which are connected directly or indirectly with one MGW form a domain or subnet. This domain or subnet can be seen as a service provider's IP network in implementation.

The WMRs can connect with each other within a certain range to forward the traffic to or from the Internet for the MNs. The MNs can only connect to the WMRs to access the Internet using two types of links. One is the IEEE 802.11e link with high data rate and small coverage area, the other is the IEEE 802.16e link with 


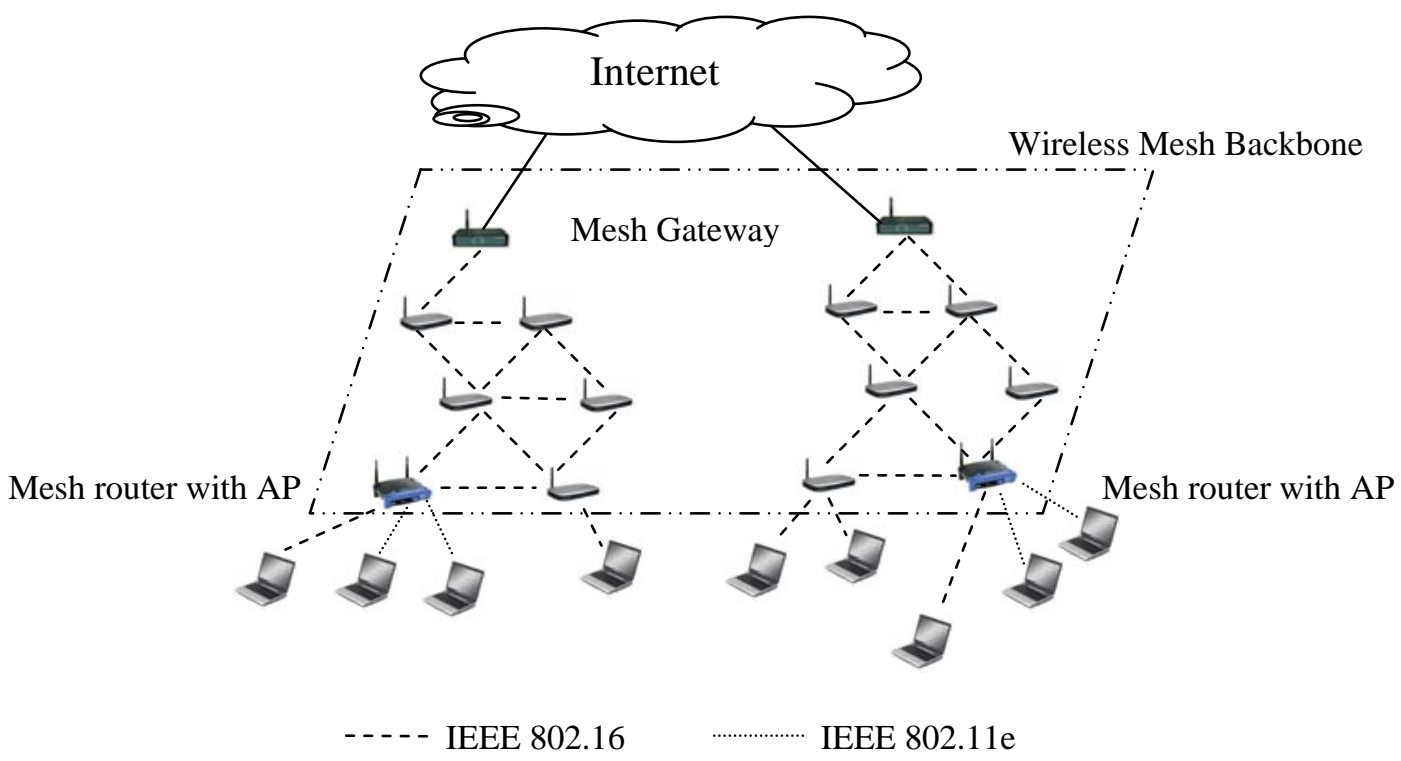

Figure 3.1: The system architecture of wireless mesh backbone.

higher data rate and larger coverage area than the IEEE 802.11e link. The IEEE 802.16e link between an MN and a WMR operates in the point-to-multipoint (PMP) mode. The IEEE 802.16e link between adjacent WMRs operates in the mesh mode. Therefore, a WMR can be seen as an integration of 802.11e AP function, 802.16e BS function with PMP mode, routing function and 802.16e subscriber station (SS) function with mesh mode. Figure 3.2 illustrates the framework of a WMR and its protocol stack. Not all the WMRs have all the above four functions. The routing function and $802.16 \mathrm{e}$ SS function with mesh mode are the basic functions of a WMR. The 802.11e AP function and 802.16e BS function with PMP mode are optional. For example, some WMRs have only these two functions and they can forward the traffic only in the mesh backbone but cannot communicate with the MNs. They can be seen as the traditional routers with a wireless interface. It is out of the scope of this thesis how to make the links between the WMRs work efficiently.

An MN initially sets up a connection using the 802.11 link or 802.16 link with the WMR. IP packets from the MN are first transmitted to the WMR. The WMR forwards the IP packets to the MGW through one or more WMRs. The MGW 


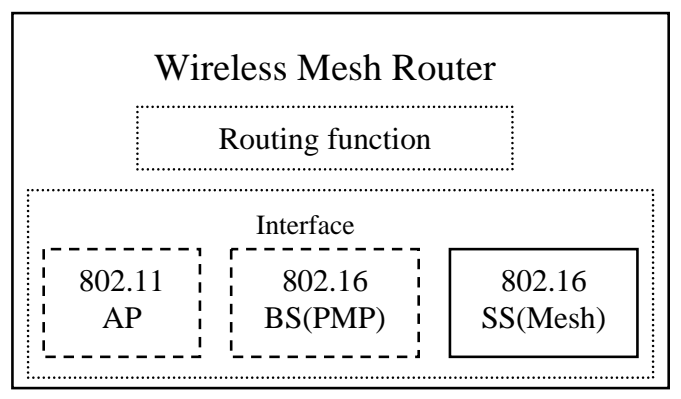

\begin{tabular}{|c|c|c|}
\hline \multicolumn{3}{|c|}{ Network Layer } \\
\hline $\begin{array}{c}802.11 \\
\text { MAC }\end{array}$ & $\begin{array}{c}802.16 \\
\text { MAC(PMP) }\end{array}$ & $\begin{array}{c}802.16 \\
\text { MAC(Mesh) }\end{array}$ \\
\hline $\begin{array}{c}802.11 \\
\text { PHY }\end{array}$ & $\begin{array}{c}802.16 \\
\text { PHY(PMP) }\end{array}$ & $\begin{array}{c}802.16 \\
\text { PHY(Mesh) }\end{array}$ \\
\hline
\end{tabular}

Figure 3.2: The functions of the WMR and its protocol stack.

uses the wired backbone to transmit the IP packets to the $\mathrm{CN}$ in the Internet. IP packets from the $\mathrm{CN}$ are routed through the reverse route to the $\mathrm{MN}$. The $\mathrm{CN}$ may be located in an administrative domain the same as the MN. Thus in this case the WMRs forward the IP packets for them. Since the coverage area of the WiMAX is larger than that of a WLAN, in the area with WLAN coverage both the Wi-Fi and WiMAX accesses are available. We call this area as double-coverage area. In this area, a proper vertical handoff for a particular MN is needed when the network is congested or when the MN is roaming across the edge of the WLAN coverage. Through proper vertical handoff, QoS support can be provided to as many as possible users. Thus, the overall resource utilization of the integrated network can be maximized. In the next section, several handoff scenarios are presented in terms of location and movement of the MN.

\subsection{Mobility Scenario}

There are several handoff scenarios due to the different mobility type of the MN. The macro-mobility and micro-mobility are described in Figure 3.3. There are two domains which are administrated by service providers A and B respectively. The WMRs in the same domain are called Intra Mesh Routers. The WMRs in different domains are called Inter Mesh Routers. For example, WMR4 and WMR5 are Intra Mesh Routers and WMR2 and WMR3 are Inter Mesh Routers in Figure 3.3. The handoff procedure between Inter Mesh Routers is more complex than that between 
Intra Mesh Routers. If the $\mathrm{CN}$ is located in an administrative domain the same as the MN, the IP packets between the MN and the CN are routed by Intra Mesh Routers and are not going through the Internet. When the MN moves to another domain, the IP packets from the $\mathrm{CN}$ can be routed to the HA and the HA then forwards them to the new location of the MN. The details will be discussed in our proposed handoff scheme in Section 4.1.

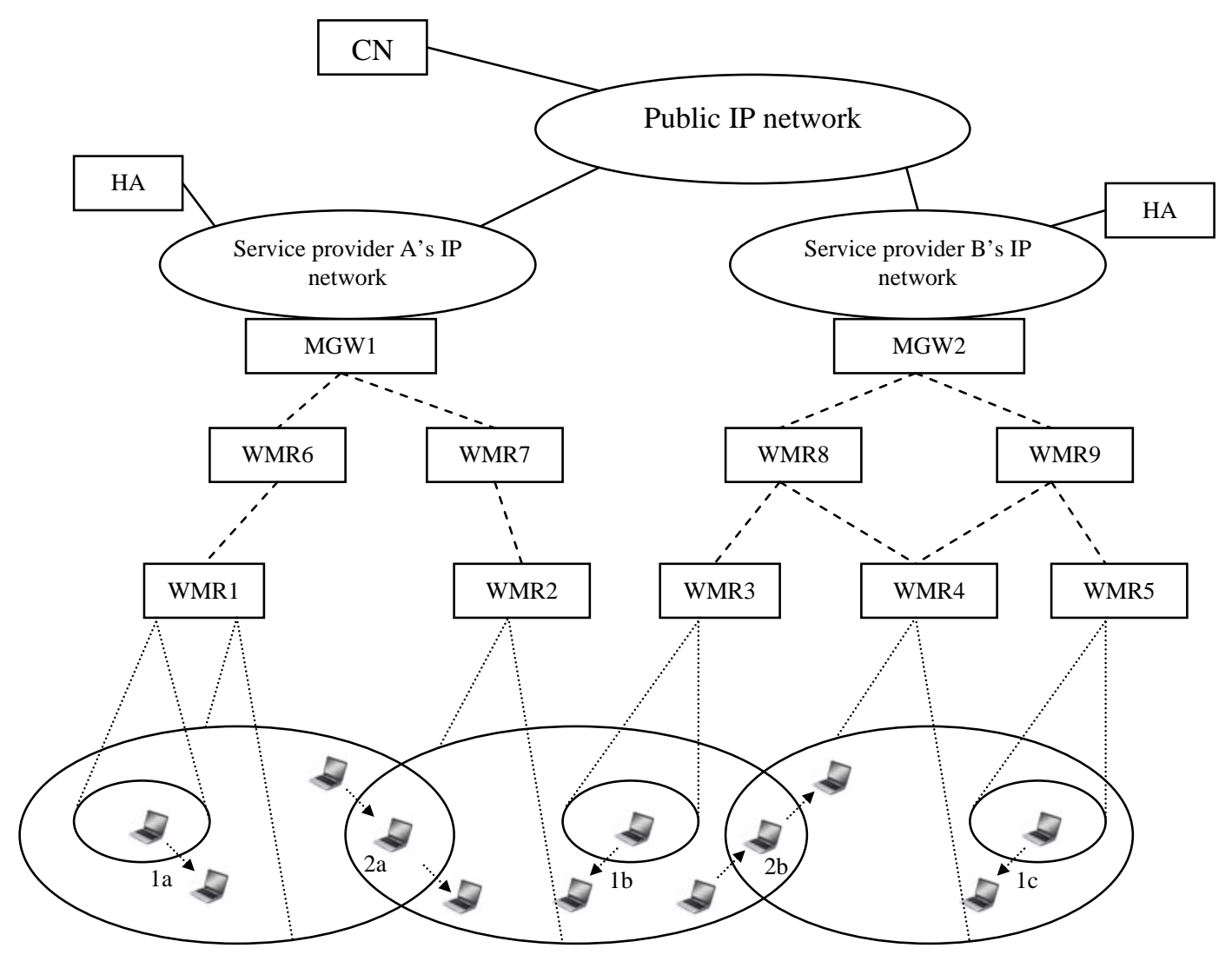

Figure 3.3: The macro-mobility and micro-mobility scenario.

Scenario 1: The MN is currently connected via a WLAN. When the MN is moving out of the coverage of the WLAN, it should change to connect to the WiMAX network because of the degradation of the signal strength from the WLAN. The movements 1a, 1b and 1c in Figure 3.3 belong to this situation. The WMR does not change and only the medium 
access interface changes in case 1a. The handoff occurs between Intra Mesh Routers in case 1c and between Inter Mesh Routers in case 1b.

Scenario 2: The MN is currently connected with the WiMAX network. When the MN moves into the WLAN coverage, it can change to connect to the WLAN or keep the connection with the WiMAX depending on the network conditions, user preference, or application requirements.

Scenario 3: The MN is located in the double-coverage area and may be stationary. When the WLAN is congested, if new traffic arrives at an MN in the WLAN, the MN can switch to the WiMAX network if it can provide more bandwidth for the MN to transmit the new traffic.

Scenario 4: The horizontal handoff happens when the MN moves in case 2a and 2b in Figure 3.3. In [19], a loss-free handoff scheme called Last Packet Marking (LPM) is proposed for case 2a. Through LPM, the MAC and network layer handoff procedures are done simultaneously to minimize the handoff time and eliminate the packet losses. The MIPSHOP (Mobility for IP: Performance, Signaling and Handoff Optimization) working group of the Internet Engineering Task Force (IETF) has been working on the Mobile IPv6 fast handoff over 802.16e networks for case $2 \mathrm{~b}$ [20].

The IEEE 802.21 working group [21] has been working on standard development to enable handoff between heterogeneous networks including both 802 and non-802 networks. It helps with handover initiation, network selection, and interface activation during vertical handoffs by introducing a Media Independent Handoff Function (MIHF). The MIHF provides multiple services such as link layer triggers and Media Independent Information Service. By using the link layer triggers, it is to minimize the connectivity disruption during link switching. Information Service can help with network discovery and selection, leading to more effective handover decisions. The authors in [22] present an extended 802.21 framework with new functions for 
network selector policy and QoS adaptation. These are high level frameworks. In this thesis, we focus on the vertical handoff for the $\mathrm{MN}$ between the Wi-Fi and WiMAX access networks based on the wireless mesh backbone architecture as shown in Figure 3.3. The vertical handoff decision and signaling procedure will be discussed in details in Chapter 4.

\subsection{QoS Support}

There are two types of wireless links between the MN and the WMR in Figure 3.3: 802.11e and 802.16e. Due to the different technologies and media access control (MAC) mechanisms, the QoS support of these two links is also different.

\subsubsection{QoS in WLAN}

The IEEE 802.11 standards are the most common standards for wireless local area networks. A WLAN supports data rates up to 54 Mbps at a range of about 30 to 300 m. It is originally designed for data transfer to provide a best effort service. There are two main medium access coordination functions defined in IEEE 802.11 MAC layer: the basic Distributed Coordination Function (DCF) and the optional Point Coordination Function (PCF). The DCF is a distributed medium access mechanism based on carrier sense multiple access with collision avoidance (CSMA/CA). It uses a contention-based MAC protocol as the basic access mechanism and cannot guarantee delay and throughput due to its probabilistic nature. As a result, the realtime applications may suffer arbitrarily long time delay and low throughput. The PCF is an optional centralized access control method in the IEEE 802.11 MAC, which is designed only for infrastructure network configurations. Although the contention-free service is designed to provide QoS for real-time traffic, this service presents some limitations and problems. Requirements of real-time traffic cannot be satisfied in general. The main limitations include the unpredictable beacon delay and unknown transmission time of polled stations [23]. 
The IEEE 802.11e Working Group [24] defines a medium access mechanism in order to enhance the QoS support of the original 802.11 MAC standard, which is called hybrid coordination function $(\mathrm{HCF})$. The QoS enhancements are available to QoS stations (QSTAs) associated with a QoS access point (QAP). The HCF combines functions from the DCF and PCF to provide QSTAs with prioritized and parameterized QoS access to the wireless medium. The HCF uses both a contention-based channel access method, called the enhanced distributed channel access (EDCA) mechanism, for contention-based transfer and a controlled channel access, referred to as the HCF controlled channel access (HCCA) mechanism, for contention-free transfer. EDCA inherits all the contention schemes and parameters of the original 802.11 DCF and provides service differentiation through prioritized access to the wireless medium. Priority service is supported through the introduction of four Access Categories (ACs) as listed in Table 3.1. Each AC has its own transmit queue, and an independent medium access function called enhanced distributed channel access function (EDCAF), with a set of AC parameters. The parameters include the arbitration interframe spacing (AIFS), $\mathrm{CW}_{\min }$ and $\mathrm{CW}_{\max }$. Higher priority ACs adopt lower values for AIFS and $\mathrm{CW}_{\min }$ to gain a higher probability of successful medium access. The values of AIFS, $\mathrm{CW}_{\min }$ and $\mathrm{CW}_{\max }$ for each $\mathrm{AC}$ in a non-AP QSTA can be obtained by using the information in the most recent EDCA Parameter Set information element of Beacon frames received from the QAP. With proper tuning of these parameters, the performance of delay sensitive multimedia traffic can be improved.

Table 3.1: Access Category

\begin{tabular}{|c|c|c|}
\hline Priority & AC & Designation \\
\hline Lowest & AC_BK & Background \\
\cline { 2 - 3 } $\mid$ & AC_BE & Best Effort \\
\cline { 2 - 3 } $\mid$ & AC_VI & Video \\
\cline { 2 - 3 } Highest & AC_VO & Voice \\
\hline
\end{tabular}


IEEE 802.11e provides differentiated services using the HCF. As delay sensitive multimedia traffic has higher priority to access the medium, the performance of the multimedia traffic using the EDCA can be clearly improved comparing with the original DCF mechanism. However without admission control and resource allocation, providing QoS guarantees only by differentiating flows and coordinating the order of channel access cannot be effective under high traffic loads. Therefore, the EDCA mechanism cannot provide guaranteed QoS and the multimedia traffic cannot be protected. Centrally controlled medium access scheme using the HCCA can provide guaranteed QoS in some sense via a polling mechanism, since a polled TXOP according to the QoS requirement is assigned to the QSTA from the QAP. Also the beacon frame delay problem is solved by the constraints of the length of the TXOP in the HCCA [25]. As compared with the EDCA, the HCCA is more complex and inefficient for normal data transmission. It also leads to expensive cost to be implemented. It is still a challenge to guarantee QoS at the contention-based MAC layer.

\subsubsection{QoS in IEEE 802.16}

The IEEE 802.16 standard [26] is designed to provide broadband wireless access with QoS provisioning. It supports data rates up to $130 \mathrm{Mbps}$. There are two modes for sharing the wireless medium in the 802.16 standard. One is the pointto-multipoint (PMP) mode, in which a BS serves a set of subscribers stations (SSs) within the same antenna sector in a broadcast manner. All the SSs receive the same transmission from the BS and the transmissions from the SSs are directed to and centrally coordinated by the BS. The other one is mesh mode and it is optional. In the mesh mode, traffic can be routed through other SSs and can occur directly between SSs.

In the IEEE 802.16 standard, uplink (from SS to BS) and downlink (from BS to SS) data transmissions are frame-based. In the downlink subframe, the transmission is broadcast and all the SSs listen to the PDU transmitted by the BS. The SSs retain 
only those PDUs addressed to them. In the uplink subframe, the SSs share the medium in a Time Division Multiple Access (TDMA) manner. Different duplexing techniques are supported for the downlink and uplink subframes. In Frequency Division Duplex (FDD), the uplink and downlink channels are located on separate frequencies. A SS can work either in full-duplex, i.e., it can transmit and receive simultaneously or half-duplex, i.e., it can receive in the downlink channel only when it is not transmitting in the uplink channel. In Time Division Duplex (TDD), the uplink and downlink transmissions occur at different times and share the same frequency.

The MAC protocol in IEEE 802.16 is connection-oriented. All data communications including both transport and control are in the context of a unidirectional connection. At the beginning of the downlink subframe, the downlink map (DLMAP) and uplink map (UL-MAP) messages are broadcasted periodically. These two messages contain the start and the end time of the downlink and uplink grants for the SSs. The BS controls the access to the medium. The bandwidth is requested by an SS on a connection basis and the uplink bandwidth is allocated by the BS to an SS as a whole. An SS has to implement locally a scheduling algorithm to redistribute the granted capacity to all of its connections [27]. There are several bandwidth request mechanisms: unsolicited requests, unicast polls, broadcast/multicast polls and piggybacking.

The 802.16 MAC specifies four different scheduling services in order to meet the QoS requirements of multimedia applications: unsolicited grant service (UGS), realtime polling service (rtPS), non-real-time polling service (nrtPS), and best effort (BE). Each scheduling service is characterized by a mandatory set of QoS parameters, which is tailored to best describe the guarantees required by the applications that the scheduling service is designed for. Furthermore, for uplink connections, it also specifies which mechanisms to use in order to request bandwidth. To effectively improve throughput while maintaining QoS guarantee, including especially delay performance and fairness, the call admission control and scheduling schemes 
are both important. However the 802.16 standard does not provide details of them and leaves to the manufactures.

\subsection{Problem Statement}

The vertical handoff occurs between different networks. The process of deciding and executing a vertical handoff is different from that in a horizontal handoff. It can be divided into three steps. At first, the MN should search and find a new available wireless network or receive the service advertisements which are broadcasted by different wireless technologies. Then the MN will select one of the multiple interfaces associated with different wireless technologies to communicate with others through the most appropriate network. Finally the vertical handoff will be executed through interactive signaling to make sure that the traffic is transmitted to the right destination.

Our main work of this thesis is how to implement the three steps above between WLAN and WiMAX in the different mobility scenarios in Figure 3.3. In the network discovery phase, the different methods will lead to different discovery time and power consumption. In the handoff decision phase, we will study how to make a decision according to the different mobility scenarios and different traffic conditions of the current network. We will also study detailed signaling in MAC and IP layer in the handoff execution procedure to control the route of the traffic, in order to avoid packet loss and reduce the handoff delay. The vertical handoff procedure can help to implement call admission control in directing a new call to the other network. 


\section{Chapter 4}

\section{Proposed Vertical Handoff Scheme}

In general, the handoff procedure consists of two stages: Layer 2 (Link layer or L2) handoff and Layer 3 (IP layer or L3) handoff. L2 handoff is the actual transfer of the radio connection between two different network interfaces. L3 handoff is to support the L2 handoff by performing packet buffering and rerouting. The handoff procedure is completed by the L2/L3 interaction. In order to maintain satisfactory QoS for IP traffic, packet delay and loss during the handoff need to be minimized. In the traditional handoff, the L3 handoff is started after the L2 handoff has been done and this leads to a large time period during which the $\mathrm{MN}$ is unable to send or receive packets. Mobile IPv6 Fast Handover Protocol (FMIPv6) [28] has been proposed as a mechanism to improve the handoff latency by predicting and preparing an impending handoff in advance. Comparing with horizontal handoff, the MN with different network interfaces can keep the previous link during the vertical handoff procedure unless the previous link is indeed broken. In other words, if the vertical handoff trigger is initiated properly, the vertical handoff procedure can be finished prior to the time when the previous link is indeed disconnected due to the movement of the MN. Our proposed vertical handoff scheme uses some L3 messages in [28] to implement L3 handoff. There are several vertical handoff 
scenarios due to the different mobility scenarios of the MN in our system model in Figure 3.3. The vertical handoff procedure is different for the different handoff scenarios especially for the L3 handoff.

In our system model in Figure 3.3, the MNs do not change their IP addresses when they move within the same domain. IP packets addressed to an MN are routed to the WGW through the Internet and then to the MN through the Intra Mesh Router. The routing table of the Intra Mesh Routers is illustrated in Table 4.1. It is an example of WMR1 in Figure 3.3. The WMR checks the destination IP address of a received packet to look up the next-hop to which the WMR should forward the packet. The third column indicates the physical interface which the WMR should use to forward the packet. If the destination IP address is not in the table list, the WMR shall use the default next-hop to forward the packet. The "N/A" of the next-hop means that the node with the destination IP address is currently associated with the WMR through the physical interface indicated in the third column.

Table 4.1: Routing table of the WMR1

\begin{tabular}{|c|c|c|}
\hline Destination & Next-Hop & PHY \\
\hline Default & WMR6 & 802.16 Mesh \\
\hline WMR6 & N/A & 802.16 Mesh \\
\hline MN1 & N/A & 802.11 \\
\hline MN2 & N/A & 802.16 PMP \\
\hline$\ldots$ & $\ldots$ & $\ldots$ \\
\hline
\end{tabular}

When an MN changes the physical interface to communicate with its $\mathrm{CN}$, the MN should get the information of the access router associated with the new physical interface to complete the L3 handoff. In [28], the MNs know the new access routers information by using the "Router Solicitation for Proxy Advertisement (RtSolPr)" and "Proxy Router Advertisement (PrRtAdv)" messages through the previous access router. The method by which the access routers exchange information about their neighbors and obtain the information about neighboring subnets to send the PrRtAdv message to the MN is not presented. In [29] a process called "candidate 
access router discovery" (CARD) is introduced to allow MNs to resolve the L2 address of one or more attach points to the IP address of the associated candidate access routers (CARs) to which an MN has a choice when performing L3 handoff. It also allows the access routers to populate and maintain their local CAR table which is an L2-L3 mapping table to resolve the L2 address of the new interface to the IP address of the associated CAR. This address-mapping table is configured statically or populated dynamically for the CARD protocol operation. In our proposed handoff scheme, we assume that all the WMRs in the wireless mesh backbone support fast handoff in [28] and each edge WMR with which the MN can connect directly already has a complete information list of the neighboring edge WMRs which have overlapping coverage areas. This list contains the edge WMR's IP address, the HA's IP address, the different interfaces' L2 addresses and the new network prefix information (NNPI). The HA belongs to the network where the corresponding WMR is located. It is used for the L3 handoff during the handoff between Inter Mesh Routers. The NNPI is for the generation of the new CoA of the MN. Table 4.2 is an example of the information list in the edge WMR4 in Figure 3.3.

Table 4.2: Information list of the neighboring edge WMR

\begin{tabular}{|c|c|c|c|c|}
\hline $\begin{array}{c}\text { IP address } \\
\text { of WMR }\end{array}$ & $\begin{array}{c}\mathbf{8 0 2 . 1 1} \text { AP } \\
\text { L2 Address }\end{array}$ & $\begin{array}{c}\mathbf{8 0 2 . 1 6} \text { BS } \\
\text { L2 Address }\end{array}$ & $\begin{array}{c}\text { IP address } \\
\text { of HA }\end{array}$ & $\begin{array}{c}\text { New Network } \\
\text { Prefix Information }\end{array}$ \\
\hline WMR3 & AP3 & - & HA & NNPI_B \\
\hline WMR4 & - & BS4 & HA & NNPI_B \\
\hline WMR5 & AP5 & - & HA & NNPI_B \\
\hline$\ldots$ & $\ldots$ & $\ldots$ & $\ldots$ & $\ldots$ \\
\hline
\end{tabular}

\subsection{Handoff Procedure}

The whole signaling procedure of vertical handoff can be divided into four stages: (1) New network interface scanning; (2) New access router discovery; (3) New network entry; (4) Updating routing information. After these stages, the MN can transmit or receive information data packets through the new network interface. 


\subsubsection{New network interface scanning}

If the MN is currently connected with the WMR using an IEEE 802.11 link, the MN shall scan the possible channels of the downlink frequency band of operation until it finds a valid downlink signal. Through the DL-MAP, DCD, UCD and UL-MAP MAC management messages, the MN can achieve MAC synchronization and obtain the downlink and uplink parameters [26]. The DCD and UCD messages contain burst profiles which determine physical layer characteristics. The DL-MAP and ULMAP messages contain the burst allocation decided by the BS. These four messages are generated by the BS periodically. The standard [26] defines the maximum DCD interval and the UCD interval to 10 seconds. The delay of the downlink and uplink synchronization is the most significant (order of magnitude is in seconds) [30]. It will slow down the network entry process of the 802.16 link.

If the MN is currently connected with the WMR using an IEEE 802.16 link, the MN shall perform scanning to acquire the AP information. There are two types of scanning mode: passive scanning and active scanning. In passive scanning, the MN shall listen to each channel scanned for no longer than a maximum duration defined by the Channel Time parameter. Active scanning involves the generation of Probe frames and the subsequent processing of received Probe Response frames. The delay in scanning dominates the total link-layer handoff time [31].

Whether the MN performs scanning to obtain new 802.11 AP or 802.16 BS information, the scanning delays are too large to be tolerant especially for real-time traffic. The author in [31] assumes that the MN always stays in the WiMAX coverage and the WiMAX interface changes to the sleep mode when the MN switches the connection to WLAN. When the MN wants to switch back, the network re-entry process is unnecessary to restore the data connection. However it does not count the situation in which the information changes in the DCD and UCD messages during the sleep mode period of the WiMAX interface. The simplest method is to keep all interfaces of the MN always on. However the activated interfaces consume the battery power without sending or receiving any packets. There is a tradeoff 
between the power consumption and obtaining accurate scanning information. To avoid degrading handoff performance, it is better for the handoff initial trigger to be started after the network interface scanning stage. In our handoff scheme, we assume this stage has been done before the handoff initial trigger occurs.

\subsubsection{New access router discovery}

Once a new BS or AP is detected through the new network interface scanning, the MN tries to learn the WMR information. With the new 802.16 BSID or 802.11 APID (L2 address of the interface), the MN requests the new WMR (NWMR) information from the currently associated WMR. In general the currently associated WMR is also called previous WMR (PWMR) in handoff procedure. The RtSolPr and PrRtAdv messages of FMIPv6 [28] are used for the resolution. The MN may send an RtSolPr as a response to some link-specific event (handoff initial trigger) or simply after performing new network interface scanning.

The MN sends an RtSolPr to its PWMR to resolve one or more AP's associated router information. In response, the PWMR sends a PrRtAdv message containing the NWMRs IP address and the HAs IP address which are searched in the information list in Table 4.2. The HA's IP address is a new option type we define for the PrRtAdv message in [28]. This HA is of the network which the NWMR belongs to. When the MN receives the PrRtAdv message which includes a HA's IP address the same as its own, it means that the MN is trying to handoff between Intra Mesh Routers in the same domain and the IP address of the MN should not be changed during the handoff. When the MN receives the PrRtAdv message which includes a different HA's IP address from its own, it means that the MN is trying to handoff between Inter Mesh Routers in the different domains and a new CoA should be

assigned to the MN to make sure that the MN can send and receive packets in the new domain network after the handoff. The updating routing information procedure is different according to the different handoff scenarios and will be discussed in Section 4.1.5. 


\subsubsection{New network entry}

In general, the new network interface scanning is a part of the new network entry process. According to the different effect to the vertical handoff, we put the new network interface scanning to an individual stage. In this stage, it is mainly concerned that how the link layer connection is set up after the new network interface scanning and a handoff decision. The signaling processes in this stage are all defined in the standards $[24,26]$.

\section{Setting up 802.16 link}

After the MN achieves MAC synchronization and obtains the downlink and uplink parameters, the ranging process can be performed to acquire the correct timing offset and power adjustments through RNG-REQ and RNG-RSP messages. The MN also can obtain the basic connection identifier (CID) and primary management CID. Immediately after completion of ranging, the MN informs the BS of its basic capabilities by transmitting an SBC-REQ message with its capabilities set to "on". The BS responds with an SBC-RSP message with intersection of the capabilities of both the MN and the BS set to "on". Following the process of authorization and key exchange, the registration process is performed by the REG-REQ and REGRSP messages so that the MN can enter the network and receive its secondary management CID and become manageable.

\section{Setting up 802.11 link}

After the MN performs a scan to find the available APs, the MN joins one of the APs to synchronize its physical and MAC layer timing parameters according to the handoff decision. Then the MN requests authentication with the AP. The MN sends an association request to the AP. When the association request frame is received from the $\mathrm{MN}$ and the $\mathrm{MN}$ is authenticated, the AP transmits an association response with a status to the MN. When the status of success is acknowledged by 
the $\mathrm{MN}$, the $\mathrm{MN}$ is considered to be associated with the AP.

\subsubsection{Updating routing information}

\section{Updating route between Intra Mesh Routers}

In [28] there are two modes of L3 handoff operation. One is the predictive mode in which updating routing messages are sent through the previous access router's link before the new connection link is set up. This mode causes some packets being buffered or lost in the new access router during the link setup time between the MN and new access router. The other mode is the reactive mode in which updating routing messages are sent through the new access router's link after the new connection link is set up. Also this mode causes some packets being buffered or lost in the previous access router during the time interval from the beginning of the disconnection of the previous link to the time when the previous access router receives the fast binding update message. The time interval, in which that the MN cannot receive packets either in the predictive mode or in the reactive mode, includes the L2 handoff and L3 handoff times. However in the case of vertical handoff between Intra Mesh Routers, since the MN has two independent network interfaces and the IP address of the MN would not be changed, it still can receive packets during the L2 handoff unless the previous link is broken. Therefore in our scheme we choose the reactive mode to operate the vertical handoff. After the new link layer connection is set up, the L3 handoff will be executed through the Updating Routing Information (URI) message.

The URI message contains a routing list (the MN's IP address, Next-Hop, PHY interface). Next-Hop is the sender's IP address of the URI message which is sent through the PHY interface. First the URI message is sent by the MN to the NWMR. After receiving the URI message the NWMR updates its routing table. If there is no record for the MN in NWMR's routing table, the list in the URI message will be added to the routing table, and after that the NWMR will send an URI message 
to its default Next-Hop about the MN's route. If there is a record for the MN in NWMR's routing table, that record will be updated by the list in URI message and after that the NWMR will send a URI message to the previous Next-Hop for the $\mathrm{MN}$ in the routing table. After the WMR in the backbone receives a URI message and updates its routing table about the MN, the WMR will send a URI message following the above rules until the URI message reaches the PWMR. When the PWMR receives the URI message, it only updates its routing table according to the routing list in the URI message and forwards the packet whose destination is the MN to the new route if there are these kinds of packets in its buffer. The PWMR may send an L3 handoff finish conform message to the MN through the previous link.

Note that admission control during handoff decision has been performed. For example, a service flow is created between the MN and NWMR by using DSA-REQ, DSA-RSP and DSA-ACK messages [26] according to the QoS requirement when the new link is 802.16 based. Therefore after the updating routing information stage is done, the packets from the $\mathrm{CN}$ to the MN can be transmitted to the MN through the new link connection.

\section{Updating route between Inter Mesh Routers}

The procedure in this situation is more complicated than that in the preceding situation because the MN is assigned the CoA and a new route must be established to this new CoA. With the information provided in the PrRtAdv message, if there is a different between the HA's IP address of the MN and that of the candidate network for handoff, the $\mathrm{MN}$ formulates a prospective CoA which is not verified yet. For instance, the prospective CoA can be generated by simply combining the network prefix and the L2 address of the MN in a stateless address autoconfiguration.

After the handoff decision is made, the MN sends the Fast Binding Update (FBU) message to its HA. We add one more option which is the new networks 
HA address (FA address) in the FBU message [28]. In response to the FBU, the HA establishes a binding between the Home Address and the proposed CoA of the MN, and sends the Fast Binding Acknowledge (FBack) to the MN. Prior to establishing this binding, the HA should send a Handover Initiate (HI) message to the FA to verify the proposed CoA. The FA sends a Handover Acknowledge (HAck) in response. The FA has a list of all nodes on its subnet and by searching this list it can confirm whether the proposed $\mathrm{CoA}$ is a duplicate. If the proposed $\mathrm{CoA}$ is not accepted by the FA, a new CoA is assigned by the FA and indicated in the HAck message. The HA should update the new CoA in the binding cache. After receiving the HAck, the HA sends FBack message which should include the new CoA to inform the change to the MN. The result of the FBU and FBack processing is that the HA begins tunnelling the packets (whose destination is the MN's Home address) to the CoA of the MN. After sending the FBack to the MN, the HA may send the URI message to the WGW to update the routing table for the MN. The rules of the sending URI message are the same in the case of updating route between Intra Mesh Routers. Until the PWMR receives the URI message for the MN, the updating routing process is not completed. This process can guarantee other MNs in the previous network can communicate with the MN which is roaming to a new network. The other MNs even do not realize the movement of the MN because they still use the Home Address of the MN to communicate with the MN.

After the L2 handoff is completed, the MN sends the URI to the NWMR to update the routing table for the $\mathrm{CoA}$ in the new network until the URI reaches to the FA. The purpose of this process is to establish the route between the MN and the FA, and to allow the FA to consider that the $\mathrm{MN}$ is reachable. If the MN does not receive the FBack message from the HA, for instance if the MN moves out of the coverage area of the previous network, the MN does not know whether the proposed CoA is verified or not. The MN sends a Duplicate Address Check (DAC) message to the NWMR to request verification for the CoA with the corresponding L2 address of the MN. When the NWMR receive this request, it first checks the $\mathrm{CoA}$ in its routing table and then sends request to the FA. If there is already an 
address the same as the CoA in the routing table, the NWMR sends a request for a new CoA for the MN to the FA. After receiving the new CoA from the FA, the NWMR sends it to the MN to update its binding cache with the new CoA. If there is no address the same as the CoA in the routing table, the NWMR sends a check request to the FA with the proposed CoA and the corresponding L2 address of the MN. By searching the list of all nodes on its subnet, the FA can confirm whether the proposed CoA is a duplicate. If the proposed CoA is a duplicate, a new CoA will be assigned by the FA. The FA sends the confirmation with results of "no duplicate" or "new CoA" to the NWMR. The NWMR informs the MN of the result. After the MN gets the verified or new CoA, it sends the URI to the NWMR to update the routing table for the CoA in the new network until the URI reaches to the FA. After the route between the MN and the FA is established, the MN can receive packets from the CN. From this time, the packets from the CN are forwarded to the MN by the HA. The MN may send a binding update message to the $\mathrm{CN}$ to set up a binding cache so that they can communicate directly. The purpose of sending a binding update message to the $\mathrm{CN}$ is to optimize the route [7]. If the $\mathrm{CN}$ is located in the same previous domain of the $\mathrm{MN}$, it is not necessary to send the binding update message to the CN. The URI message has set up the route for packets to the $\mathrm{MN}$, and the $\mathrm{CN}$ located in the same previous domain even does not realize the movement of the MN.

\subsubsection{Example of handoff signaling procedure}

\section{Case 1: Vertical handoff within a WMR}

This case happens during the movement 1a in Figure 3.3. The MN just changes the network interface to establish an 802.16 link with the same WMR. Therefore the route of the packets from the $\mathrm{CN}$ to the $\mathrm{MN}$ is only changed between the WMR and the MN. It is not changed between the $\mathrm{CN}$ and the WMR. Figure 4.1 simply illustrates the route change and the signal control. When a handoff trigger is initiated, the MN sends an RtSolPr message to WMR1 to obtain the 


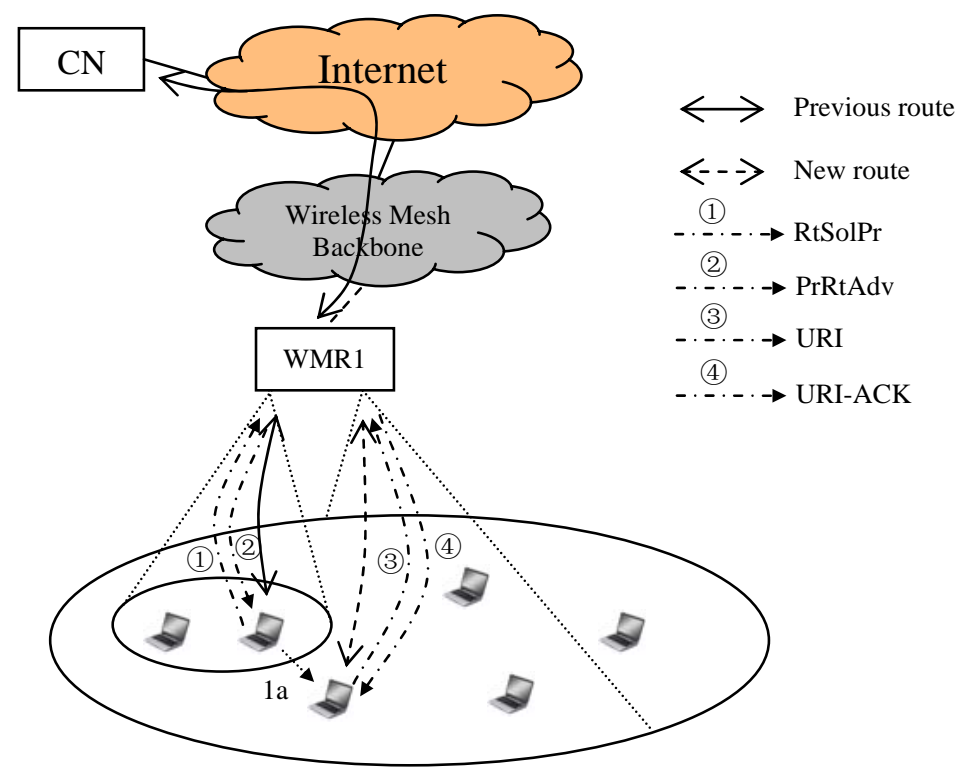

Figure 4.1: Route change and signal control in vertical handoff with the same WMR.

new 802.16 BS's associated routers information. The L2 address of the new 802.16 BS is obtained in the new network interface scanning stage. After receiving the RtSolPr message, WMR1 sends the response PrRtAdv message to inform the MN that the associated router of the new $802.16 \mathrm{BS}$ is WMR1. After that, the MN sets up the 802.16 link with the new 802.16 BS. Note that the L2 signaling used is in section 4.1.3 and it is not displayed in Figure 4.1. When the 802.16 link is successfully set up, the MN sends the URI message to WMR1 to update the routing information from (MN's IP address, N/A, 802.11) to (MN's IP address, N/A, 802.16PMP). After the MN receives an URI-ACK message which indicates the success of updating route, the MN can use the new 802.16 link to communicate with the CN.

\section{Case 2: Vertical handoff between Intra Mesh Routers}

This case happens during movement 1c in Figure 3.3. Figure 4.2 is redrawn to illustrate the vertical handoff signaling procedure for the movement. The solid line 


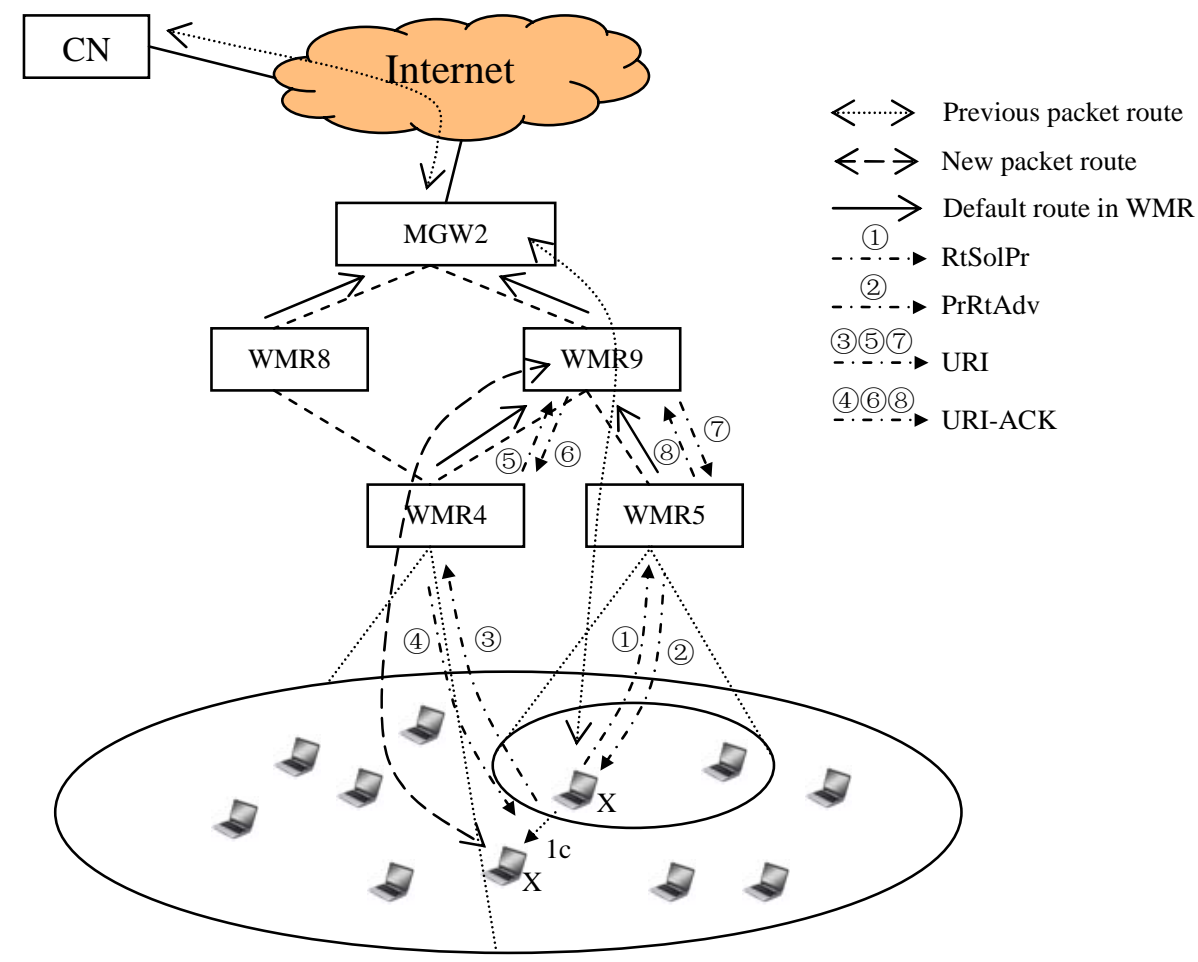

Figure 4.2: Vertical handoff between Intra Mesh Routers.

in the backbone is the default route for packets in the WMRs. For convenience, we use X to indicate the MN's home IP address. The updating route process follows the rules in section 4.1.4. The changes of the routing table are listed in Table 4.3. The mark $(\boldsymbol{V})$ indicates the changes in each node. After vertical handoff, the route of the packets communicated between the $\mathrm{MN}$ and the $\mathrm{CN}$ is changed only between WMR9 and the MN. As we can see that there is no change in the routing table in WGW2 after vertical handoff in this example. Figure 4.3 describes the details of the signaling of the handoff procedure.

\section{Case 3: Vertical handoff between Inter Mesh Routers}

This case happens during movement 1b in Figure 3.3. In this case, the MN roams into a different domain network. The different domains are administrated by different service providers. Even if the MN sets up an L2 link with the new domain 
Table 4.3: The routing table before and after the vertical handoff in case 2

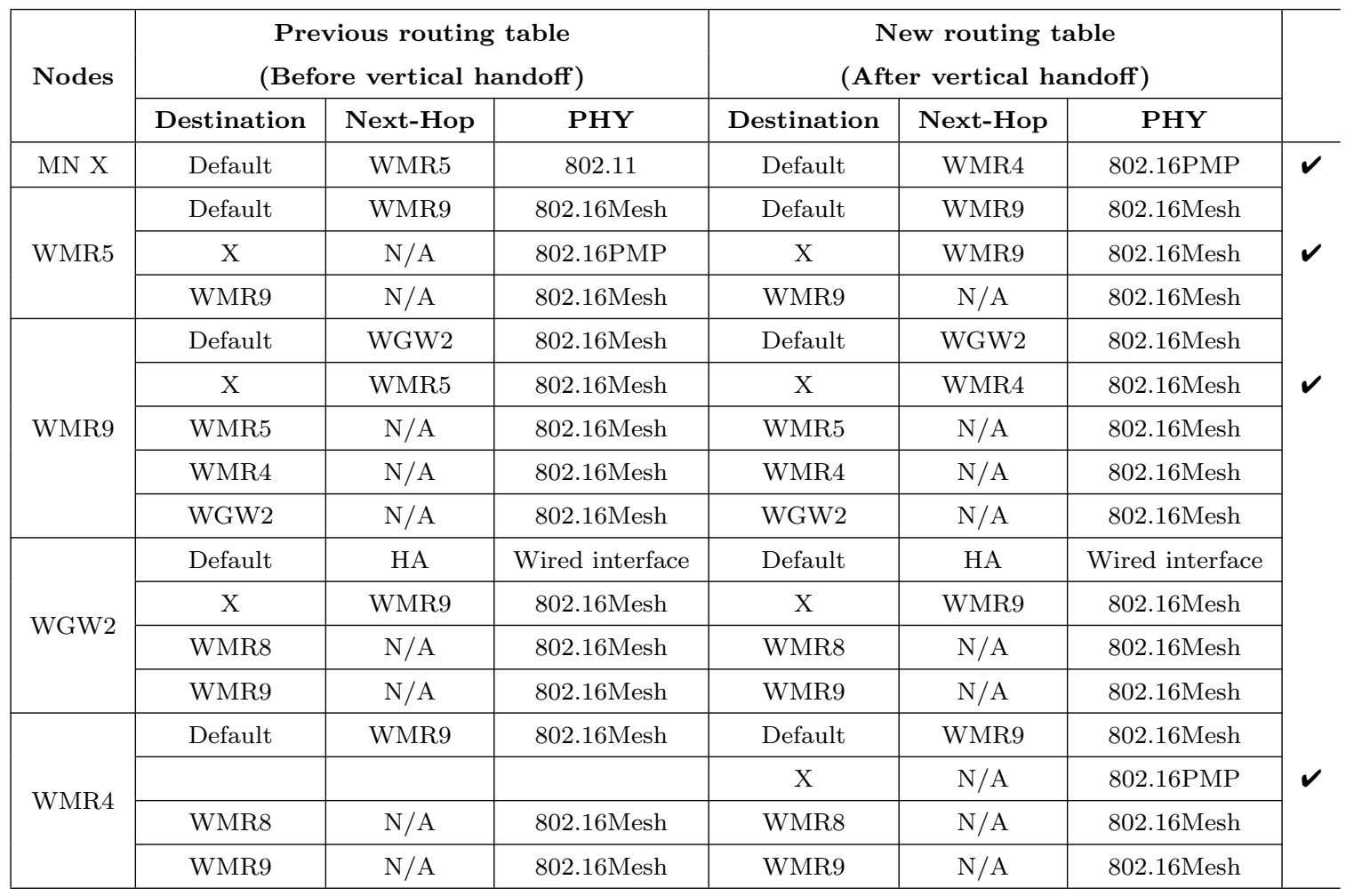

network, it still loses the IP connectivity. The Mobile IPv6 [7] provides the solution to maintain the connectivity to the Internet for the $\mathrm{MN}$ by using the CoA configuration and binding update. However the handoff latency resulting from the new CoA configuration and Binding update procedures is often large and unacceptable to real-time traffic. The fast handoff protocol in [28] is designed to reduce the above handoff latency. The messages in [28] has been used in this case for the updating routing information process when the MN roams from one domain network to another domain network. The detailed signaling procedure is illustrated in Figure 4.4 and Figure 4.5. Note it does not include the binding update from the MN to the $\mathrm{CN}$. For convenience, we use Y to indicate the MN's new CoA. The binding cache in the MN and HA is (X, Y). Table 4.4 and Table 4.5 show the changes of routing table in the new and old networks during the vertical handoff. If the MN does not receive the FBack from the HA, the MN should use the DAC and DACack messages to verify its CoA from the FA. Only after the CoA of the MN is verified by the FA, 


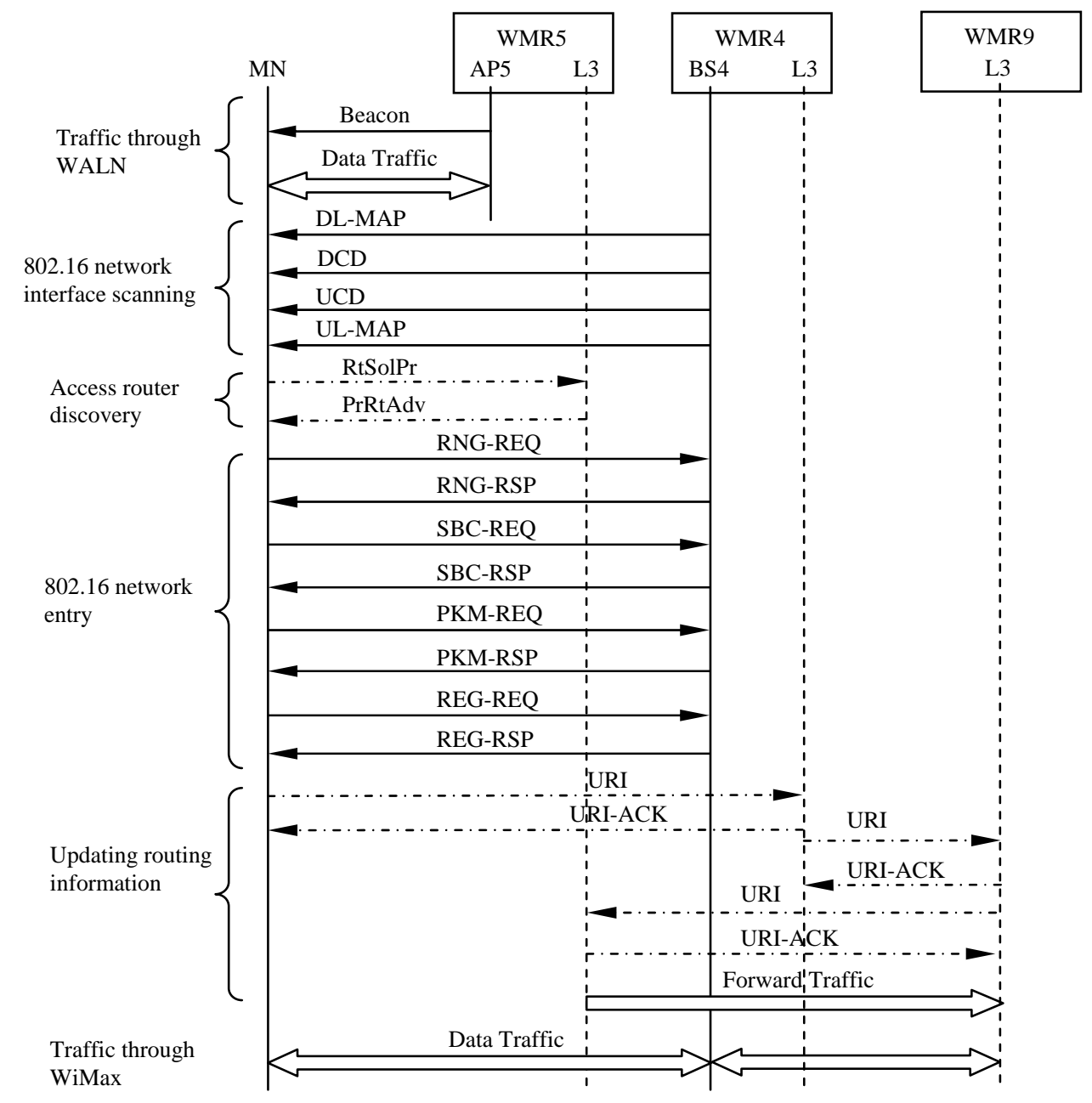

Figure 4.3: Signaling for vertical handoff from WLAN to WiMAX between Intra Mesh Routers.

will the MN use the URI message to establish the route between the FA and the MN.

\subsection{Handoff Decision}

Vertical handoff decision is an important and intelligent part of vertical handoff process. In the MN assisted vertical handoff, the MN determines which network it should connect to and when to trigger the handoff. The vertical handoff occurs when the $\mathrm{MN}$ is moving across the edge of coverage area. It is obvious that the 


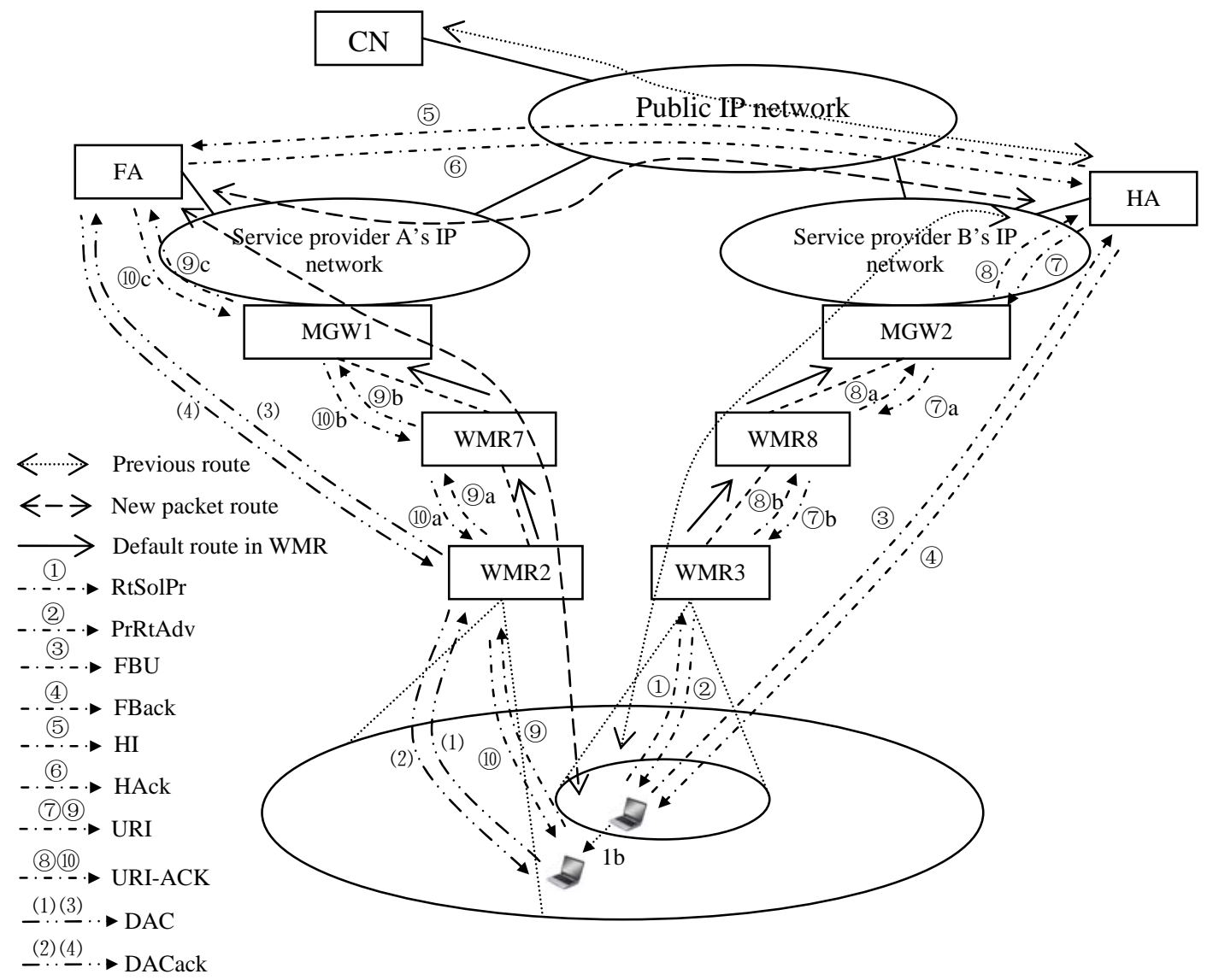

Figure 4.4: Vertical handoff between Inter Mesh Routers.

received signal strength is mainly concerned when the $\mathrm{MN}$ is moving across the edge especially from the small coverage area to the large one. In the integrated network between WLAN and WiMAX in our system model, the vertical handoff will occur when the MN crosses the edge of the different service area. The RSS of the WLAN beacons is a basic criterion. If the RSS of WLAN is the only factor used in the decision of vertical handoff, the handoff only occurs at the edge of the WLAN service area. However the vertical handoff may not take place only at the edge of service area. It can happen at any time depends on network conditions. Our goal of the vertical handoff is to provide QoS support to as many users as possible. The vertical handoff will happen at the double-coverage area even if the $\mathrm{MN}$ is not moving across the edge. Therefore in this thesis we divide the vertical handoff decision into two types. One is based on the RSS of the WLAN when the 


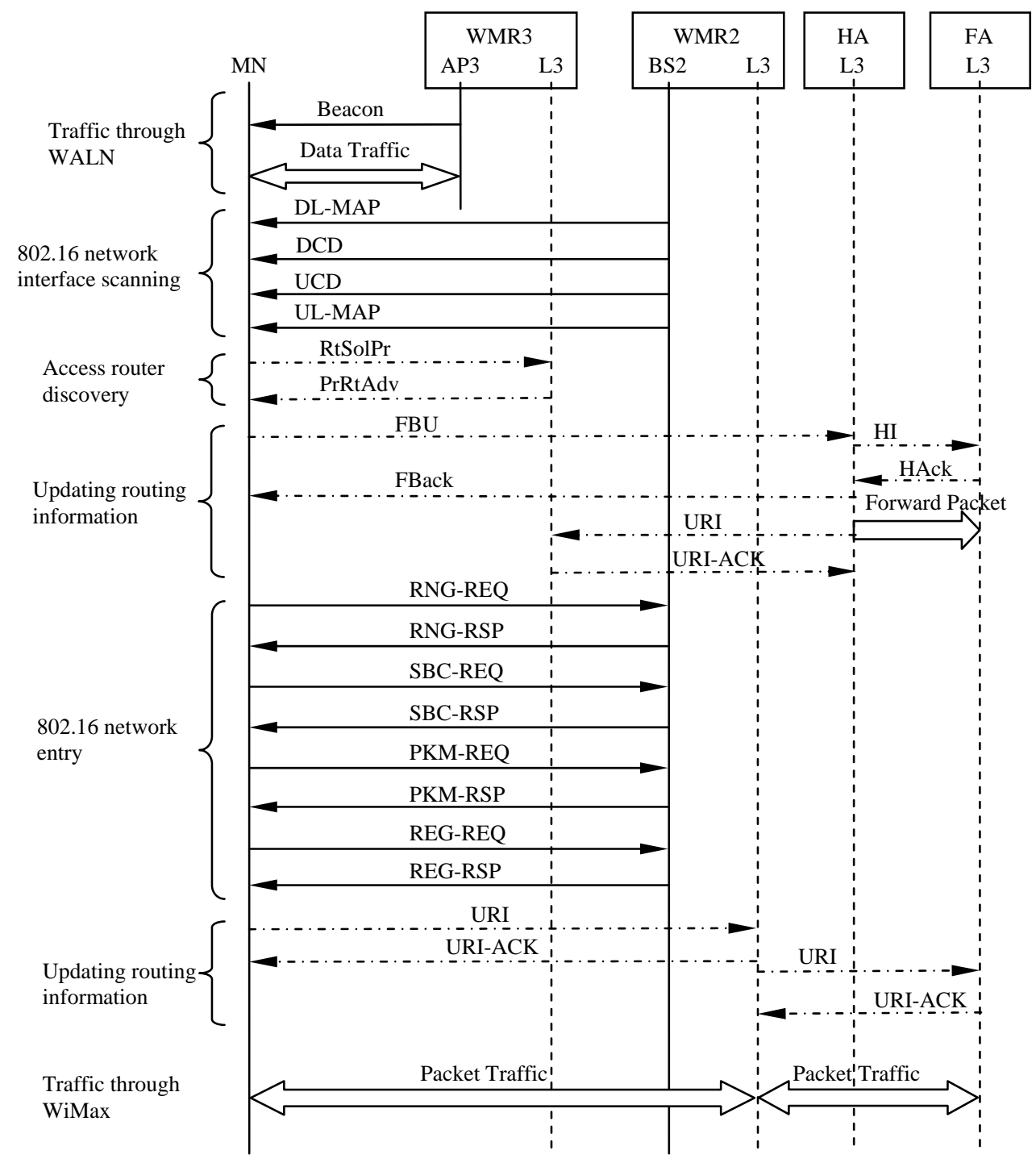

Figure 4.5: Signaling for vertical handoff from WLAN to WiMAX between Inter Mesh Routers.

MN is moving across the edge of the double-coverage area. The other is based on the network condition such as congestion when the MN is located in the doublecoverage area.

Admission control is a QoS mechanism that decides whether a new connection can be established. Also, the QoS of existing connections should not be degraded. If the new connection is rejected by the admission control mechanism, a vertical handoff process is initiated to obtain bandwidth to accommodate the new connec- 
Table 4.4: The routing table in the old network in case 3

\begin{tabular}{|c|c|c|c|c|c|c|c|}
\hline \multirow[t]{2}{*}{ Nodes } & \multicolumn{3}{|c|}{$\begin{array}{l}\text { Previous routing table } \\
\text { (Before vertical handoff) }\end{array}$} & \multicolumn{3}{|c|}{$\begin{array}{c}\text { New routing table } \\
\text { (After vertical handoff) }\end{array}$} & \\
\hline & Destination & Next-Hop & PHY & Destination & Next-Hop & PHY & \\
\hline $\mathrm{MN}(\mathrm{X}, \mathrm{Y})$ & Default & WMR3 & 802.11 & Default & WMR2 & 802.16PMP & $v$ \\
\hline \multirow{3}{*}{ WMR3 } & Default & WMR8 & 802.16Mesh & Default & WMR8 & 802.16Mesh & \multirow{3}{*}{$\checkmark$} \\
\hline & $\mathrm{X}$ & $\mathrm{N} / \mathrm{A}$ & 802.16PMP & $\mathrm{X}$ & WMR8 & 802.16Mesh & \\
\hline & WMR8 & $\mathrm{N} / \mathrm{A}$ & 802.16Mesh & WMR8 & $\mathrm{N} / \mathrm{A}$ & 802.16Mesh & \\
\hline \multirow{5}{*}{ WMR8 } & Default & WGW2 & 802.16Mesh & Default & WGW2 & 802.16Mesh & \multirow{5}{*}{$\checkmark$} \\
\hline & $\mathrm{X}$ & WMR3 & 802.16Mesh & $\mathrm{X}$ & WGW2 & 802.16Mesh & \\
\hline & WMR3 & $\mathrm{N} / \mathrm{A}$ & 802.16Mesh & WMR3 & $\mathrm{N} / \mathrm{A}$ & 802.16Mesh & \\
\hline & WMR4 & $\mathrm{N} / \mathrm{A}$ & 802.16Mesh & WMR4 & $\mathrm{N} / \mathrm{A}$ & 802.16Mesh & \\
\hline & WGW2 & $\mathrm{N} / \mathrm{A}$ & 802.16Mesh & WGW2 & $\mathrm{N} / \mathrm{A}$ & 802.16Mesh & \\
\hline \multirow{4}{*}{ WGW2 } & Default & HA & Wired interface & Default & HA & Wired interface & \multirow{4}{*}{$v$} \\
\hline & $\mathrm{X}$ & WMR8 & 802.16Mesh & $\mathrm{X}$ & $\mathrm{HA}$ & Wired interface & \\
\hline & WMR8 & $\mathrm{N} / \mathrm{A}$ & 802.16Mesh & WMR8 & $\mathrm{N} / \mathrm{A}$ & 802.16Mesh & \\
\hline & WMR9 & $\mathrm{N} / \mathrm{A}$ & 802.16Mesh & WMR9 & $\mathrm{N} / \mathrm{A}$ & 802.16Mesh & \\
\hline \multirow{2}{*}{ HA $(X, Y)$} & Default & \multicolumn{2}{|c|}{ To Internet } & Default & \multicolumn{2}{|c|}{ To Internet } & \\
\hline & $\mathrm{X}$ & WGW2 & Wired interface & $\mathrm{X}$ & \multicolumn{2}{|c|}{ Forward packets to $\mathrm{Y}$} & $v$ \\
\hline
\end{tabular}

Table 4.5: The routing table in the new network in case 3

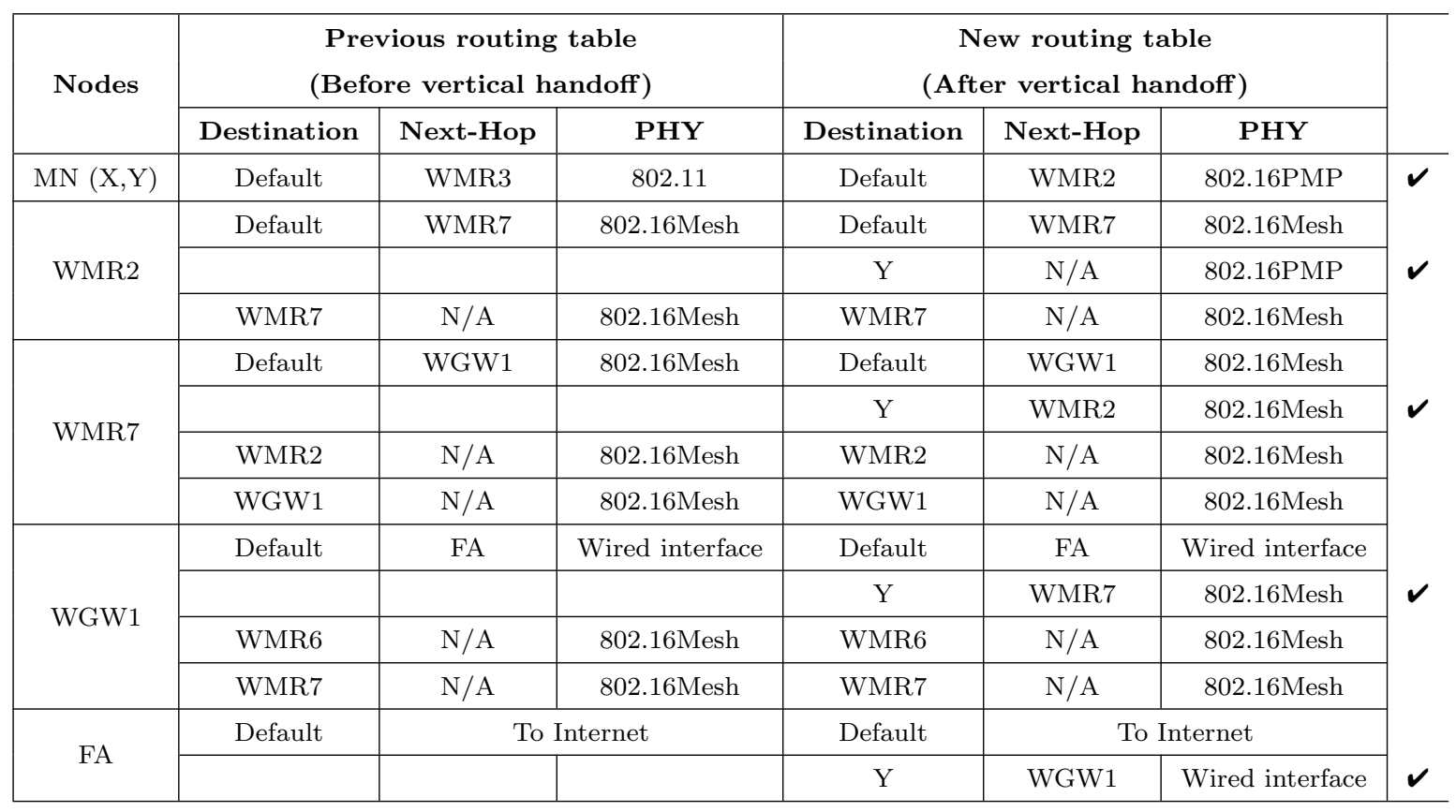

tion in the other network by the admission control. The combination of the vertical handoff and admission control can lead to our goal. 
Although the WLAN is designed originally for data transfer, a lot of research works related to the performance of the voice traffic in the WLAN have been done due to the wide deployment of the WLAN and the rapid spreading of the voice over Internet protocol (VoIP) application. The voice capacity of a WLAN, which is defined as the maximum number of voice connections that can be supported with satisfied quality, has been investigated in the literature. A typical IEEE 802.11b WLAN with $11 \mathrm{Mbps}$ bandwidth can only support a very limited number of VoIP connections in the DCF mode. In [32], the capacity of G.711 VoIP using constant bit rate $(\mathrm{CBR})$ model and a $10 \mathrm{~ms}$ packetization interval is 6 calls. The larger packetization interval leads to more capacity for VoIP connections. However a larger packetiztion interval will result in a longer delay. In [33], the voice capacity in IEEE 802.11e WLAN is studied. The occurrence of controlled access phases (CAPs) during the contention period (CP) is also considered. The results show a promising increase of the capacity of VoIP calls due to the enhanced mechanism in 802.11e. The new voice traffic above the voice capacity, without any admission control, will affect the existing voice traffic and jeopardize the network with a significant increase in delay and a decrease in throughput. In [34], an admission control algorithm for the 802.11e EDCA is proposed. By estimating the achievable throughput of each traffic flow and avoiding channel overloading, the QoS of existing flows can be maintained effectively. A measurement-based admission control scheme based on the IEEE 802.11e for differentiation service of the EDCA is proposed in [35]. For voice traffic, MNs listen to available budgets from the AP to make decisions on acceptance or rejection of a voice stream. This centrally-assisted distributed admission control provides good differentiation among different access categories and good fairness among real-time streams within the same access category. However, the decision of the admission control is made at individual MNs. The network condition information base on which the MN makes the decision is not obtained accurately since the MNs receive the information periodically and also do not know the traffic situation of other MNs. In [36] a novel call admission and rate control scheme is presented to support QoS to real-time services using the 802.11 
DCF mechanism. The channel busyness ratio, which is a measurement of the network status and is easy to be obtained, is used to represent the channel utilization accurately. An optimal operating point is found by increasing the channel busyness ratio. The maximal channel utilization corresponding to the optimal point does not change too much for different numbers of active nodes and packet size. The call admission control decision is made at the AP in the infrastructure mode. Since the traffic is going through the AP, the AP can accumulate the occupied channel bandwidth of the admitted real-time traffic flows. Based on the accurate information recorded in the AP and the maximal channel utilization, the call admission control scheme can keep the network working at the point with a maximal throughput and tolerant delay and delay jitter. This centralized admission control needs more signals to exchange information between the MN and the AP. The IEEE 802.11e standard [24] recommends admission control for the two higher ACs: AC_VO and AC_VI. When the MN receives a new real-time connection request from its application layer, the MN must send an add traffic stream (ADDTS) request message with the traffic characteristics and QoS requirements such as the mean data rate and packet size in the traffic specification (TSPEC) element to the QAP in order to request admission. Based on the network condition and new request, the QAP can use any admission control decision algorithm to decide whether or not to accept the request. After that, the QAP will send an ADDTS response message which indicates the admission result to the MN. Therefore in the integrated networks with WLAN and WiMAX, the rejected voice traffic by the admission control can be transmitted using the other network (WiMAX) if it has enough available radio resources. The result of admission control can help the MN to make handoff decision and generate a vertical handoff initial trigger. In this thesis, we use the idea from [36] and the TS set-up in [24] to implement the admission control for real-time traffic in WLAN in vertical handoff decision.

However, in [37] the simulation results show that the additional data traffic can significantly reduce the voice capacity. The mean packet delay at the AP can be very large. In other words, too many data transmissions degrade the system 
performance of the existing voice streams, because many data transmissions cause many collisions. The existing voice traffic becomes vulnerable to the data traffic. A global dynamic data parameter control mechanism is introduced to protect the existing voice traffic from best effort data traffic in [35]. In the mechanism, the AP dynamically controls best effort data parameters of MNs globally based on traffic conditions. Such a global/centralized data parameter control mechanism provides the best fairness for data transmissions among MNs. For the best effort data transmissions, the mechanism dynamically adjusts the three parameters $\left(\mathrm{CW}_{\min }\right.$, $\mathrm{CW}_{\max }$ and AIFS) of the best effort data $\mathrm{AC}$ with time. When the traffic load increases, the three parameters should be increased to reduce the collisions. When the traffic load decreases, the three parameters should be decreased to default lowest limit. Although the QoS of the existing voice traffic is protected and guaranteed through both the admission control and global data parameter control mechanism, the data traffics in a higher traffic load suffers from a very large delay. So in the integrated networks with WLAN and WiMAX, when this case happens, even if they do not have strict delay requirement, some data traffic may be transmitted using the other network (WiMAX) when the delay of the data traffic is larger than a predefined delay threshold. The handoff for some MNs with the data traffic from WLAN to WiMAX can reduce the data traffic load in the WLAN. Better performance for the data traffic can then be achieved and the resource utilization of the whole integrated network can be improved.

In the 802.16 network, the admission control and scheduling schemes are both important, the same as in WLAN. However the 802.16 standard [26] does not provide details of the admission control decision and leaves it to the manufacturers. The voice packet transmission performance using different scheduling services is studied in [38]. It shows that the UGS service achieves the best delay performance for voice traffic but with very low efficiency in utilizing the BS resources. Also, the rtPS service provides more flexibility in scheduling voice traffic. The BS can tradeoff between the packet transmission performance and resource utilization. In [39], a scheduling algorithm and an admission control policy for IEEE 802.16 standard 
are presented. A token bucket mechanism is used to admit the new connections with QoS guarantees in terms of both bandwidth and delay. A set of call admission control and scheduling schemes for real-time video traffic in IEEE 802.16 networks is proposed in [40]. The proposed CAC admits an incoming flow with more flexibility via an appropriate access time, which is figured out through coordination with existing I-frames and non-I-frames. The proposed scheduling scheme arranges realtime video packets according to individual delay performance of each flow under a loose constraint on earliest latest starting time (LST) first. In the 802.16 standard [26], an MN will send a request to the BS using a DSA-REQ message with the traffic characteristics and scheduling requirements to create a service flow, which a MAC transport service that provides unidirectional transport of packets either to uplink packets transmitted by the MN or to downlink packets transmitted by the BS. The service flow is characterized by a set of QoS parameters such as latency, jitter and throughput assurances. The admission control decision is to check whether the service flow QoS can be supported. The BS will send a DSA-RSP message to indicate acceptance or rejection. The MN will send a DSA-ACK message to acknowledge to the BS. After the service flow is set up, the service flow for the connection is mapped to MAC connection identified by a CID corresponding to the service flow. The traffic is transmitted through this connection.

Therefore, in order to provide QoS support to as many users as possible, the vertical handoff decision should be made jointly with admission control for a new call (connection). If the new connection is rejected by the current network, it should be handed off to the other network for admission. Both new and handoff calls should not violate QoS requirements of existing calls. Figure 4.6 illustrates the handoff procedure for an MN in the double-coverage area and admission control procedure for a new call of real-time traffic. If the new call cannot be admitted to WLAN, it should be transferred to WiMAX for admission using the vertical handoff procedure. 


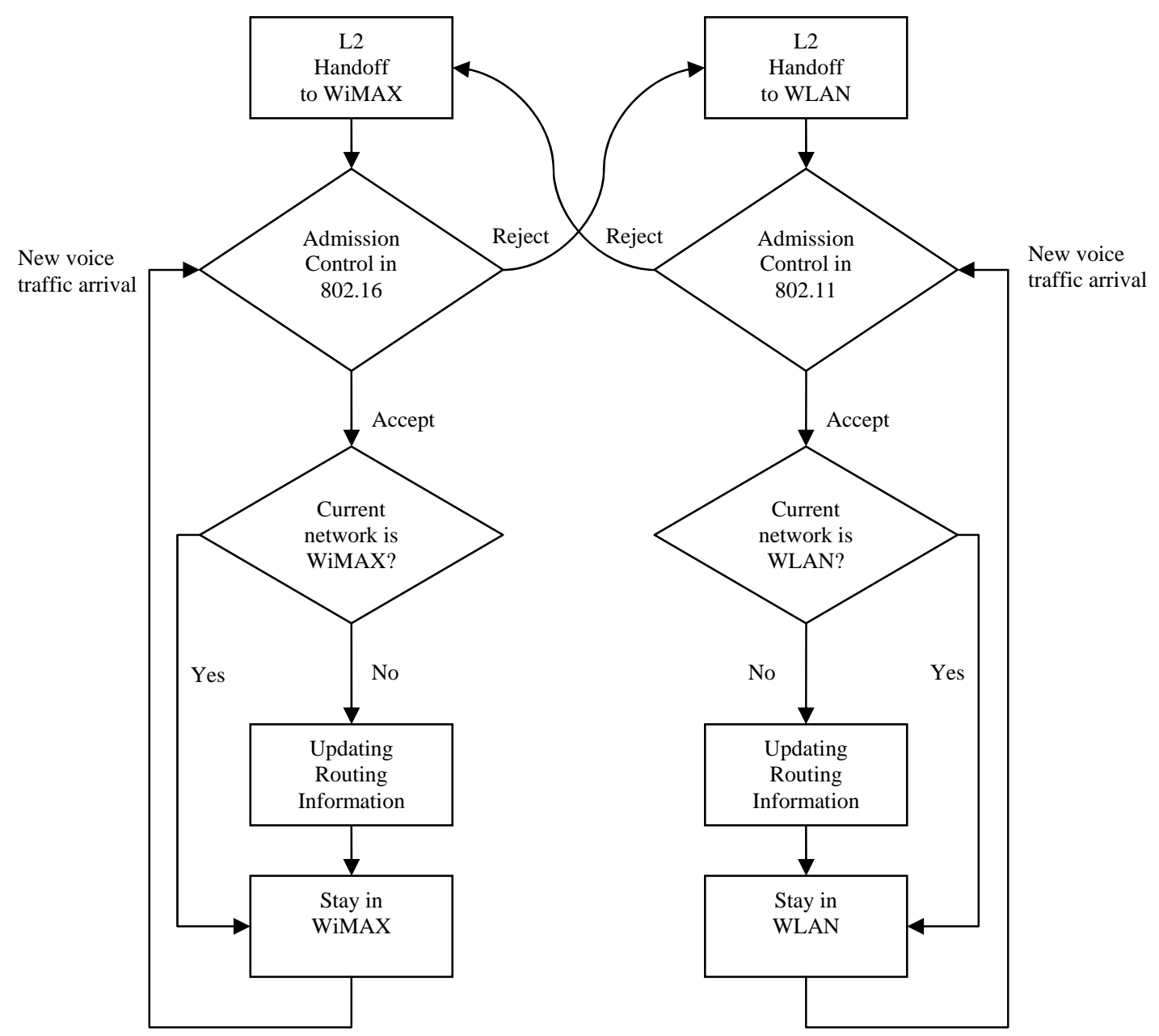

Figure 4.6: Joint admission control and vertical handoff procedure.

\subsubsection{Vertical handoff for a new call}

\section{From WLAN to WiMAX}

The MN stays in the double-coverage area and is currently connecting with the 802.11e WLAN. It receives the beacon message from the QAP periodically. From the beacon message, the MN updates the EDCA parameters for the four ACs such as $\mathrm{CW}_{\min }, \mathrm{CW}_{\max }$ and AIFS. Also the ACs (AC_VO and AC_VI) that need admission control are determined through the admission control mandatory (ACM) subfield in the EDCA parameter set element. The MN sends an ADDTS request to the QAP to request admission when a new real-time traffic call is requested from 
the upper layer. The TSPEC element in the ADDTS request includes the QoS requirements of the new real-time traffic: Nominal MSDU Size $(L)$ and Mean Data Rate $(R)$. The QAP makes the admission control decision based on the network condition and the new call requirements by using the main idea in [24] and [36]. We use the Medium Time [24] to describe the normalized bandwidth requirement of the new real-time traffic $B_{N R}$, given by

$$
B_{N R}=\frac{R}{L} \times\left(A I F S+\frac{L+H}{R_{P h}}+S I F S+\frac{A C K}{R_{P b}}\right) \times S B A .
$$

In the above equation, $H$ is the size of MAC header, $R_{P h}$ is the PHY data rate, $R_{P b}$ is the basic rate, $A I F S$ is Arbitration Interframe Space Period, SIFS is Short Interframe Space Period, $A C K$ is the packet size of ACK, $S B A$ is Surplus Bandwidth Allowance which indicates the ratio of over-the-air bandwidth to bandwidth of the transported MSDUs required for successful transmission.

In the QAP, we use $B_{u s}$ to denote the total bandwidth which has been used by the admitted real-time traffic. The maximal channel utilization $B_{U}$ of the optimal point is used to provide bandwidth for the real-time traffic. In order to reserve bandwidth for data traffic without admission control, the total bandwidth reserved for real-time traffic $B_{M}$ is a portion $(80 \%)$ of $B_{U}$. The $20 \%$ of $B_{U}$ is for data traffic without admission control. $B_{U}$ is found to be 0.9 (without RTS/CTS) or 0.95 (with RTS/CTS)[36].

After the QAP receives the ADDTS request from the MN, it calculates the normalized bandwidth requirement for the new real-time traffic and checks whether the new real-time traffic can be accommodated as the following:

If $B_{u s}+B_{N R}<B_{M}$, the QAP accepts the request and sends the ADDTS response indicating the acceptance with the specified Medium Time. Also the $B_{u s}$ will be updated to $B_{u s}+B_{N R}$. Otherwise, the QAP sends the ADDTS response indicating the rejection. Figure 4.7 describes the admission control decision algorithm in the QAP.

After the MN receives the ADDTS response with the admission result, it decides 


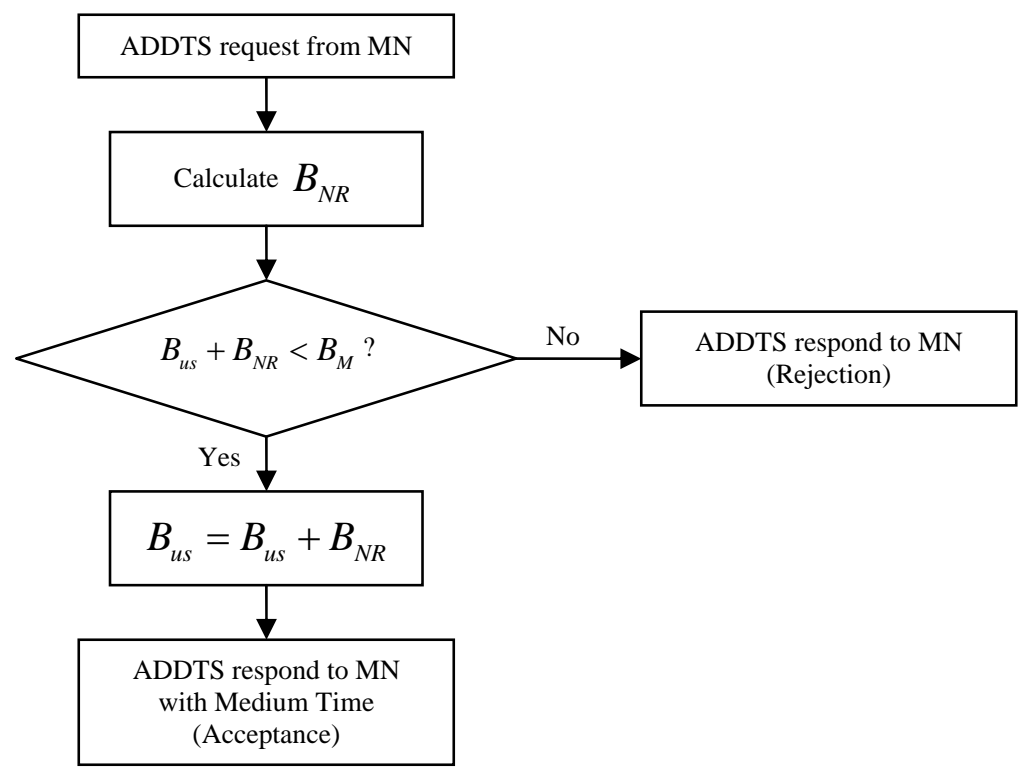

Figure 4.7: Admission control decision algorithm in the QAP.

whether to initiate an L2 handoff or not. If the request is accepted, the MN stays in the WLAN. If the request is rejected, the MN initiates an L2 handoff to the WiMAX network which is found in the new network interface scanning stage. After the L2 handoff, the MN sends a DSA-REQ message to the BS to request admission for the new traffic. Then the BS sends a DSA-RSP to the MN to indicate the admission result. If the request is accepted, the L3 handoff is triggered and the MN updates the routing information to establish an IP route to transmit the information data traffic to the $\mathrm{CN}$.

\section{From WiMAX to WLAN}

If the $\mathrm{MN}$ is currently connecting with the 802.16 network and has new real-time traffic to transmit, the vertical handoff should be initiated to provide service to the new real-time traffic if the BS is too busy to accommodate the new request. Therefore the admission control for a new connection in the WiMAX network is also helpful for the vertical handoff decision to the WLAN.

Because the MAC in IEEE 802.16 is connection-oriented, a connection or ser- 
vice flow should be set up before the information data traffic is transmitted. The admission control decision is made during the connection or service flow set-up. In [39], the admission control module is at the BS to provide QoS guarantees in terms of both bandwidth and delay for the newly and previous admitted connections. We use the main idea of this admission control module to help the MN to initiate the vertical handoff from WiMAX to WLAN. To create the new connection or service flow, the DSA-REQ and DSA-RSP messages in the standards [26] are used between the MN and the BS. The parameters in the admission control are as follows.

$C_{\text {uplink }}$ : total capacity (bps) allocated for uplink transmission.

$C_{U G S}$ : total capacity (bps) allocated for current UGS connections.

$C_{r t P S}$ : total capacity (bps) allocated for current rtPS connections.

$C_{n r t P S}$ : total capacity (bps) allocated for current nrtPS connections.

$r_{i}$ : average data rate of new service flow or connection request.

When a new connection needs to be set up, the MN sends a request to the BS using a DSA-REQ message includes the traffic characteristics such as $r_{i}$. The BS checks the available bandwidth: If $r_{i} \leq C_{\text {uplink }}-C_{U G S}-C_{r t P S}-C_{n r t P S}$, the new connection is accepted; Otherwise it is rejected. If the new request is accepted, the $C_{U G S}, C_{r t P S}, C_{n r t P S}$ values are updated according to the new connection types respectively. There is no admission control process for BE connections and they are always admitted with no QoS support. Figure 4.8 shows the admission control decision algorithm in the BS.

\subsubsection{Vertical handoff decision algorithm}

When the MN is moving out of the double-coverage area, the RSS from the AP in the $\mathrm{MN}$ is mainly concerned to start the vertical handoff. We define a threshold of

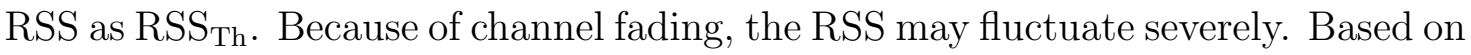
the periodic beacon message, if the MN detects the current signal strength RSS $_{\text {cur }}$ below $\mathrm{RSS}_{\mathrm{Th}}$ and then triggers the handoff, it is possible to make unnecessary 


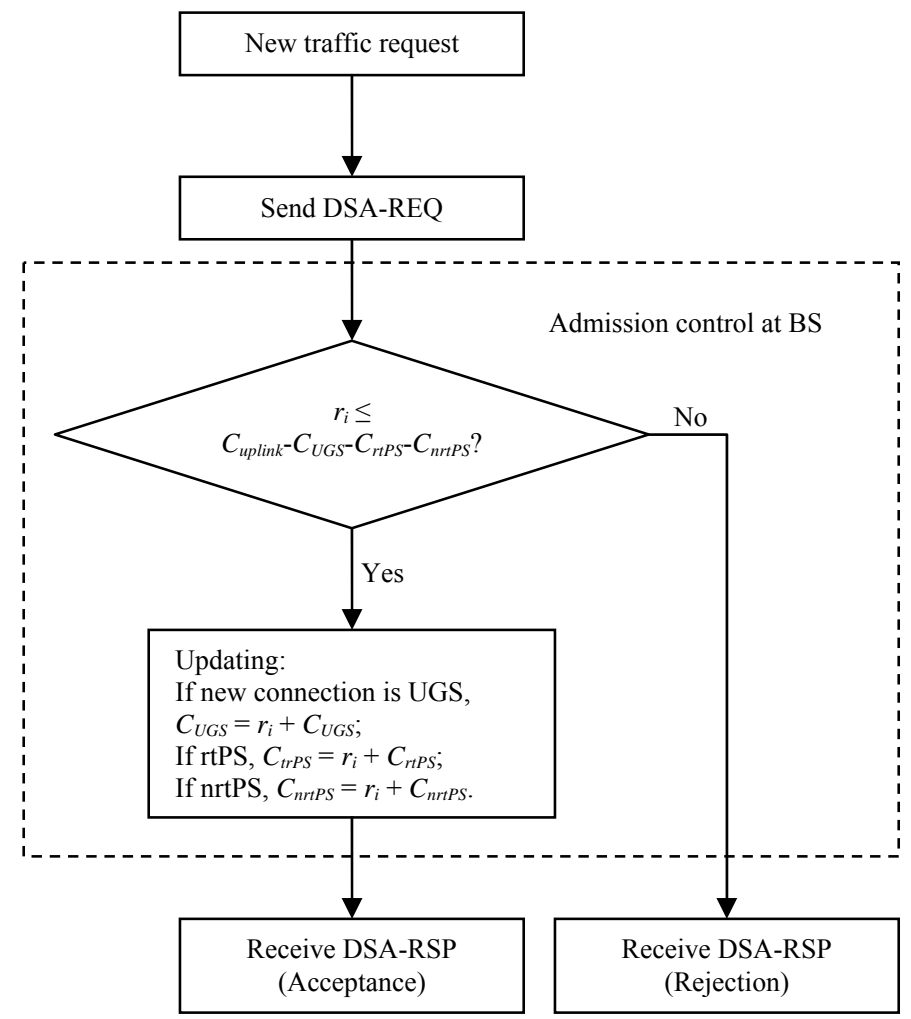

Figure 4.8: Admission control decision algorithm in BS.

handoff decision. We define a counter, $\mathrm{N}_{\mathrm{RSS}}$, to count the numbers when the $\mathrm{RSS}_{\text {cur }}$ is below $\mathrm{RSS}_{\mathrm{Th}}$. When the $\mathrm{N}_{\mathrm{RSS}}$ is large than a threshold $\mathrm{N}_{\mathrm{RSST}}$, the vertical handoff is triggered. Figure 4.9 illustrates the possible handoff decision algorithm to trigger the vertical handoff when the $\mathrm{MN}$ is moving out the double-coverage area.

In the case of vertical handoff from WiMAX to WLAN, the criterias to trigger the handoff are different from those in the vertical handoff from WLAN to WiMAX. Since the WiMAX has a larger coverage area than the WLAN, when the MN moves into the WLAN coverage area, the MN can still keep the connection with the WiMAX network. After the MN detects the existence of the WLAN, it may make a decision to trigger the handoff to WLAN depending on the user preference, the WLAN network condition and the current traffic condition in the MN. If there is current real-time traffic between the MN and BS, it is not necessary to hand off to the WLAN unless the user prefers the WLAN and also the WLAN has 


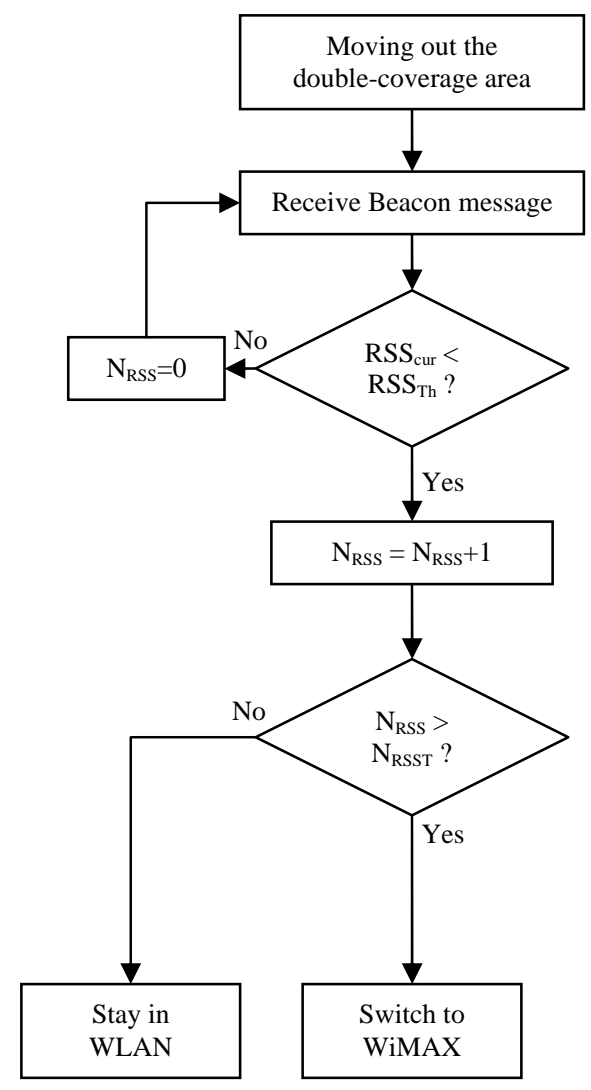

Figure 4.9: Vertical handoff decision algorithm from WLAN to WiMAX.

enough available bandwidth. To check if there is enough bandwidth for the potential handoff traffic in the WLAN, we use the concept of QBSS (QoS basic service set) load defined in the IEEE 802.11e standard [24]. The QBSS load information element is present in the beacon frame periodically generated by the AP. The QBSS load contains information about the station count, channel utilization, and available admission capacity. We use $\left(B_{M}-B_{u s}\right)$ defined in section 4.2.1 as the available admission capacity. The MN can use this information to check if there is enough bandwidth for its on-going real-time traffic. Furthermore if there is a new real-time traffic in the $\mathrm{MN}$ and the $\mathrm{BS}$ is too busy to accommodate the new request, the vertical handoff should be initiated to provide service to the new real-time traffic by using the method and procedure in section 4.2.1. Figure 4.10 shows the handoff decision algorithm to trigger the vertical handoff when the MN is moving in the double-coverage area. 


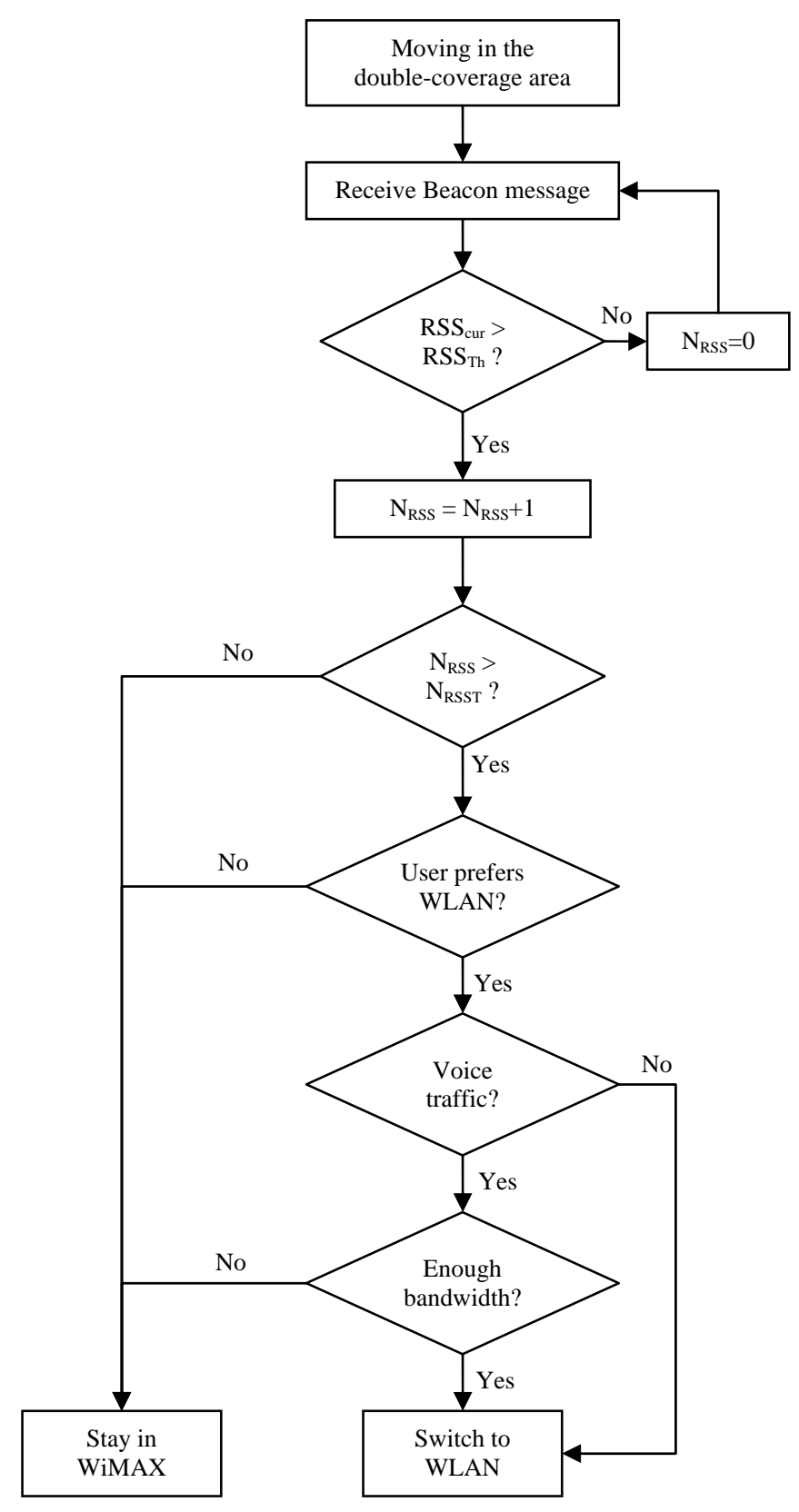

Figure 4.10: Vertical handoff decision algorithm from WiMAX to WLAN.

\subsection{Summary}

In this chapter, an vertical handoff scheme between 802.11 and 802.16 in the system model presented in Chapter 3 is proposed. The vertical handoff occurs in three scenarios: (1) within the same WMR, (2) between Intra Mesh Routers, and (3) 
between Inter Mesh Routers. The handoff signaling procedure in these different scenarios is discussed in details to reduce the signaling overhead on the backbone and provide a low handoff delay. In order to provide QoS support to as many users as possible, the vertical handoff decision is made jointly with admission control for a new call. The rejected call in the current network is handed off to the other network for admission. The QoS of existing calls is not violated by the new and handoff calls. When the MN is moving out/in the double-coverage area, the vertical handoff decision algorithm is presented respectively. When the $\mathrm{MN}$ is moving out of the double-coverage area, the RSS from the AP in the MN is the main metric to start the vertical handoff. When the MN moves into the WLAN coverage area, the MN may keep the connection with the WiMAX network or make a decision to trigger the handoff to WLAN according to the user preference, the WLAN network condition, and the current traffic condition in the MN. 


\section{Chapter 5}

\section{Perfomance Evaluation}

In this chpater, the proposed vertical handoff scheme is evaluated through simulation using a network simulator (NS2-2.31) [41]. The 802.11e model from TKN [42] and the 802.16 model from NIST [43] are used in the simulation. The performance of the proposed vetical handoff scheme has been demostrated by the simulation results with repect to signaling cost, handoff delay, and QoS support in terms of system thoughput and packet delay.

\subsection{Simulation Configuration}

In the simulation, each MN has two independent radio interfaces. One is for 802.11e, and the other for 802.16. The model of the MN in the simulation is shown in Figure 5.1. The MN switches the network interfaces using the proposed handoff procedure. The default network interface for each MN is 802.11e. In the 802.11e model, the data rate is $11 \mathrm{Mbps}$ and the basic rate is $1 \mathrm{Mbps}$. During the simulation, the RTS/CTS mechanism is disabled with no hidden terminals. The channel of wireless link is assumed to be error free. Each MN only has one active traffic flow at a time. The control messages including the admission control request during the handoff have high priority the same as AC_VO traffic. The IEEE 802.11e parameters are summarized in Table 5.1. 


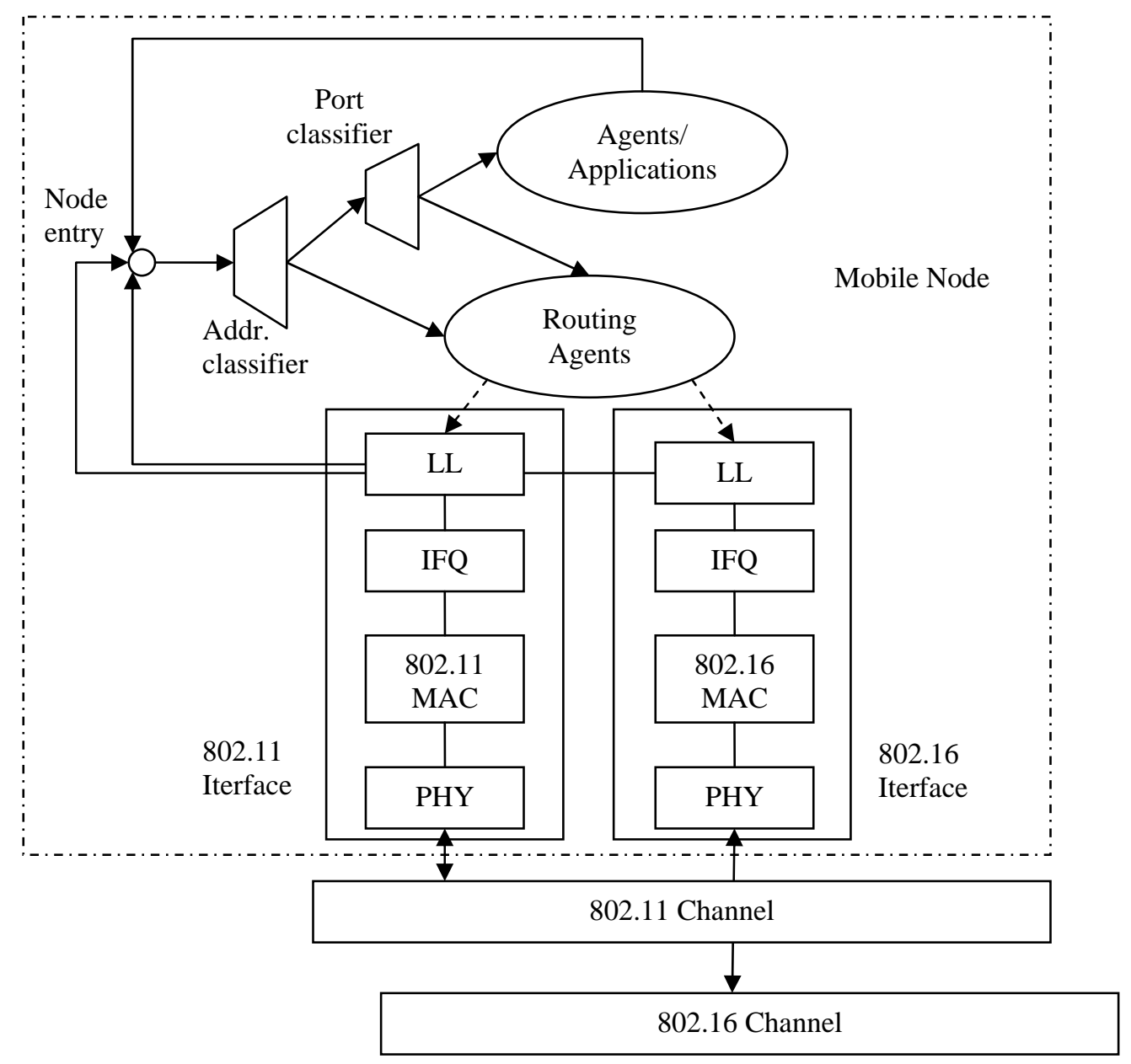

Figure 5.1: The dual-interface MN model in the simulation.

Since we focus on the performance of the vertical handoff scheme, we use the wired links instead of the wireless links in the wireless mesh backbone. The bandwidth and link delay of the wired links in the simulation are set as $100 \mathrm{Mbps}$ and $1 \mathrm{~ms}$. For each MN, there is a $\mathrm{CN}$ to establish a traffic connection. The traffic from an $\mathrm{MN}$ to a $\mathrm{CN}$ first arrives at the $\mathrm{AP}$ or $\mathrm{BS}$, then is forwarded to a gateway node, and each $\mathrm{CN}$ is connected to the gateway node directly. Hence there are 2 wired links and 1 wireless link between the MN and CN. The international standard G.711 for encoding voice traffic has a fixed bit rate of $64 \mathrm{Kbps}$. Therefore, in the simulation, the real-time traffic is modeled with a constant rate of $64 \mathrm{Kbps}$, a packet size of 160 bytes, and the packet inter-arrival time $20 \mathrm{~ms}$. Moreover, to sim- 
Table 5.1: IEEE 802.11e simulation parameters

\begin{tabular}{ll}
\hline Data rate & $11 \mathrm{Mbps}$ \\
Basic rate & $1 \mathrm{Mbps}$ \\
Phy header & $192 \mathrm{bits}$ \\
MAC header & $240 \mathrm{bits}$ \\
Slot Time & $20 \mu \mathrm{s}$ \\
SIFS & $10 \mu \mathrm{s}$ \\
AIFS & SIFS+n×Slot Time \\
$\mathrm{n}$ & AC_VO, AC_VI: 2 \\
& AC_BE: 3 \\
& AC_BE: 7 \\
\hline
\end{tabular}

plify the simulation, we do not implement the admission control request in 802.16 BS. The 802.16 BS can always accept the traffic when the MN transfers the new rejected calls by the 802.11 e network to the 802.16 network during the handoff.

To eliminate the warming-up effects, during the simulation, the first MN starts generate information packets at $50 \mathrm{~s}$, followed by other MNs one by one, where each new MN waits for additional 5 s to start transmission. In the simulation, the vertical handoff jointly with admission control for a new call can be observed.

We also simulate the vertical handoff when the MN moves in/out the doublecoverage area. First we add the traffic to each MN in the WLAN until no more traffic can be accepted by the WLAN. Then one of the MNs in the WLAN starts to move outwards from the WLAN at a speed of $10 \mathrm{~m} / \mathrm{s}$. The vertical handoff will be triggered since the RSS of that MN decreases. After staying in the WiMAX for a while, the MN moves back to its original position at the same speed. The vertical handoff will occurs again when the MN crosses the edge of the double-coverage area. 


\subsection{Simulation Results}

\section{System throughput and packet delay}

As more MNs begin to transmit their traffic, the system traffic load increases gradually with time. Initially, the 802.11 AP can accept all the new call requests. When the traffic load reaches a certain level, the admission control takes effect in rejecting new calls to ensure QoS (e.g., in terms of packet delay) of calls in service. The rejected calls are transferred to the 802.16 network. The whole procedure follows the handoff decision with admission control which is described in section 4.2.1. As shown in Figure 5.2, the maximum number of MNs that can be accepted in the 802.11 AP increases when the packet size of the traffic becomes larger with a fixed rate, $64 \mathrm{Kbps}$. Obviously with the fixed rate, the packet inter-arrival time also increases corresponding to the increasing packet size. The value of the SBA (Surplus Bandwidth Allowance) used in the simulation is 1.5.

Figure 5.3 shows the total system throughput as the traffic load increases (with time). It is observed that the $802.11 \mathrm{AP}$ can accept up to $18 \mathrm{MNs}$. This is consistent with the result in Figure 5.2. The handoff occurs to the 19th MN. As shown in Figure 5.3(a), if we do not transfer new calls via the vertical handoff procedure, the total throughput decreases immediately after the 19th MN joins the WLAN. In this case, the system cannot provision QoS to all the MNs. Figure 5.3(b) shows that all the $19 \mathrm{MNs}$ can obtain sufficient system resources for their traffic flows when the 19th MN is transferred to WiMAX and provided with QoS support in WiMAX. The data rates of the existing traffic flows are not affected by admitting the 19th MN to WiMAX via vertical handoff. We define the end-to-end packet delay as the time interval between the MN starting to send a packet and the $\mathrm{CN}$ in the Internet receiving that packet in the application layer. This packet delay includes the delay in the wired links which is a fixed value of $2 \mathrm{~ms}$ since there are 2 wired links between the MN and CN and the delay of each wired link is set to $1 \mathrm{~ms}$. Figure 5.4 shows the end-to-end packet delay of the first MN, which increases with the WLAN traffic load (time). It is observed that the delay is increased dramatically from time 140s 


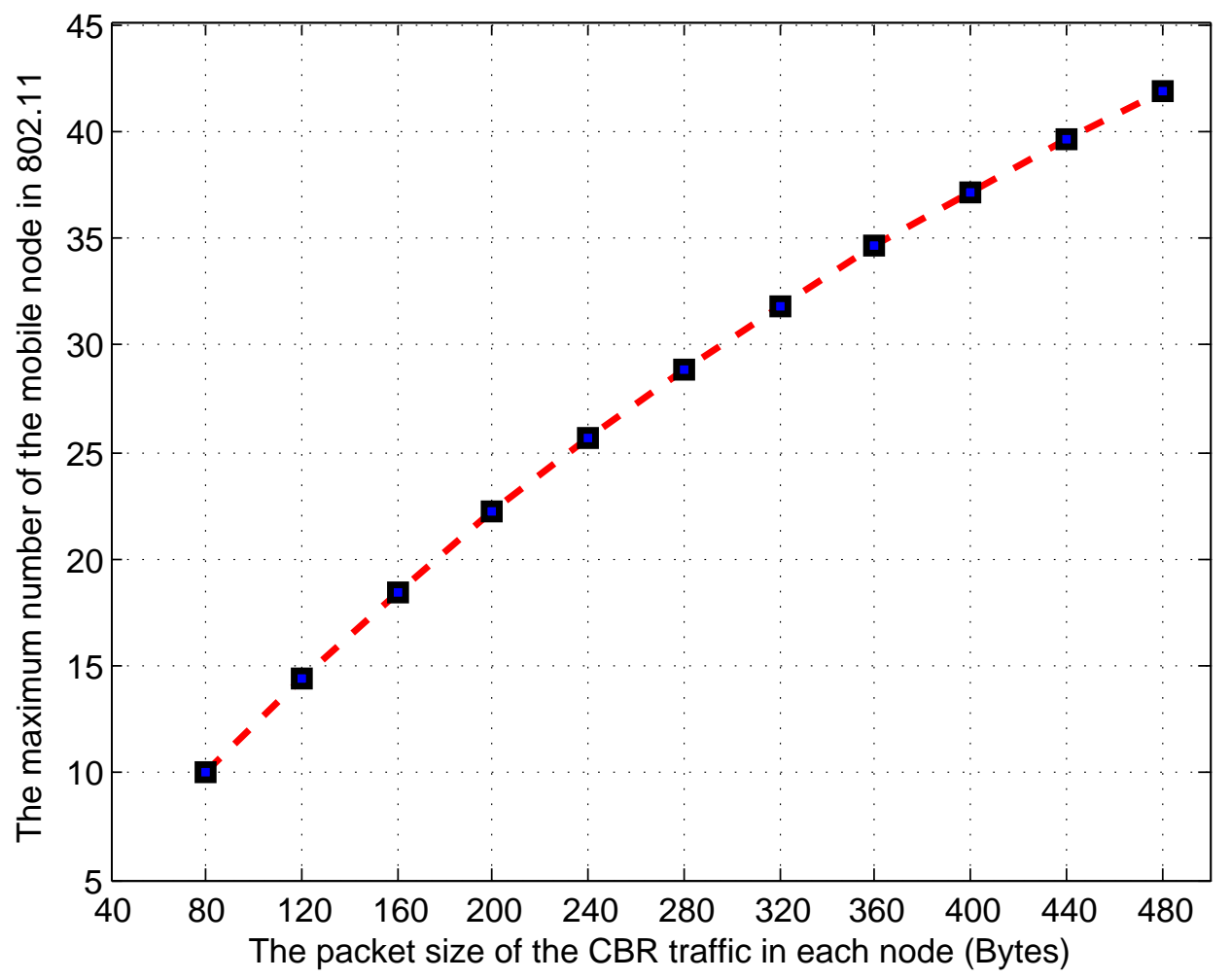

Figure 5.2: The maximum number of MN in 802.11 with QoS support.

when 19th MN joins the WLAN. On the other hand, if the 19th MN is transferred to the WiMAX, the packet delay of the first MN remains steady. With our proposed handoff scheme, the end-to-end packet delay can be kept at a low value.

The propagation model used for the AP in the simulation is two-ray ground reflection model. The received power $P_{r}$ in the MN according to the distance $d$ between the MN and $\mathrm{AP}$ is given by

$$
P_{r}=\frac{P_{t} * G_{t} * G_{r} * h_{t}^{2} * h_{r}^{2}}{d^{4} * L}
$$

In the simulation, the transmitted signal power $P_{t}$ in the AP is set to $7.214 \mathrm{~mW}$. Both the antenna gains of the transmitter and the receiver, $G_{t}$ and $G_{r}$, are set to 1. The heights of the transmit and receive antennas, $h_{t}$ and $h_{r}$, are set to 1.5. The system loss $L$ is equal to 1 . When the first $\mathrm{MN}$ is moving out/in the double-coverage area, the RSS from the AP in the MN changes as shown in Figure 5.5. When the 


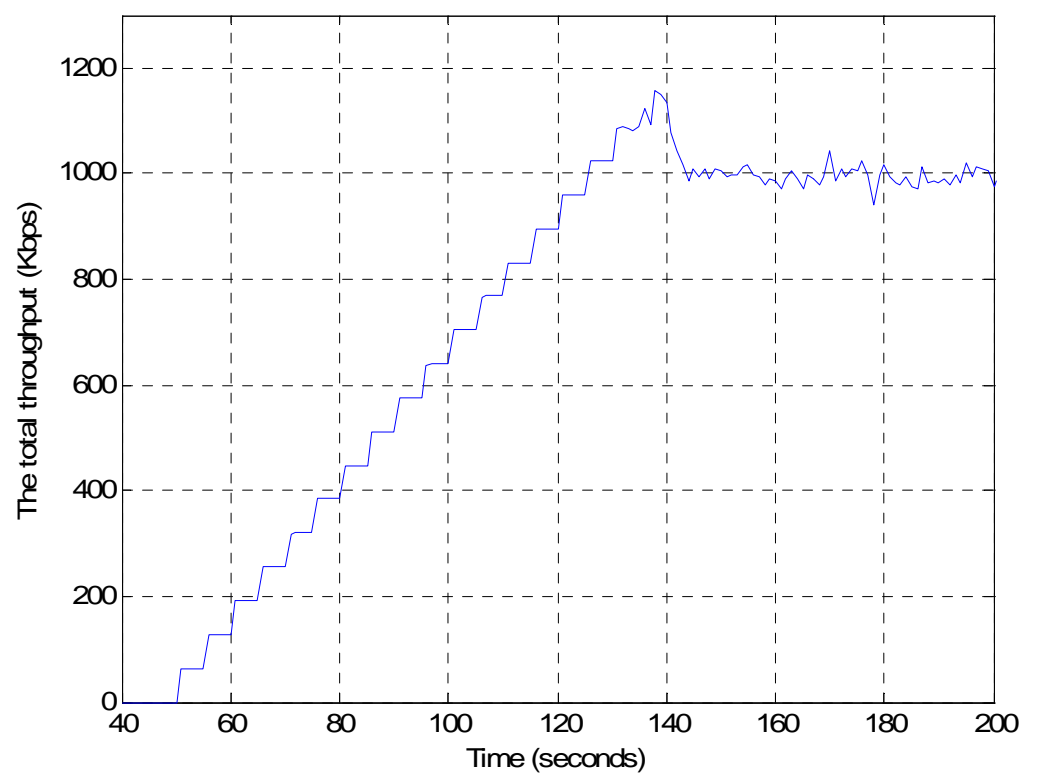

(a) Without vertical handoff.

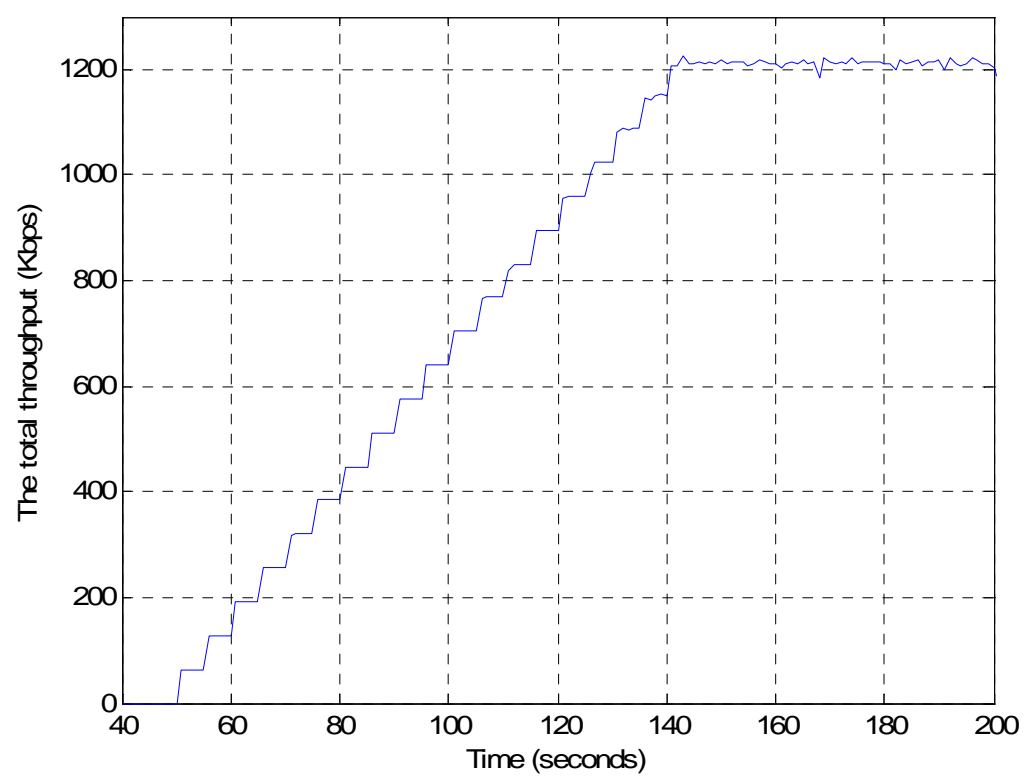

(b) With vertical handoff.

Figure 5.3: The system throughput versus traffic load.

MN is moving out and the RSS is below the threshold, the vertical handoff is trigged. Figure 5.6 shows the changes of the end-to-end packet delay of the MN due to the handoff. It is observed that the packet delay in the WiMAX link is a fixed value. This is because the MAC mechanism in WiMAX is different from the 


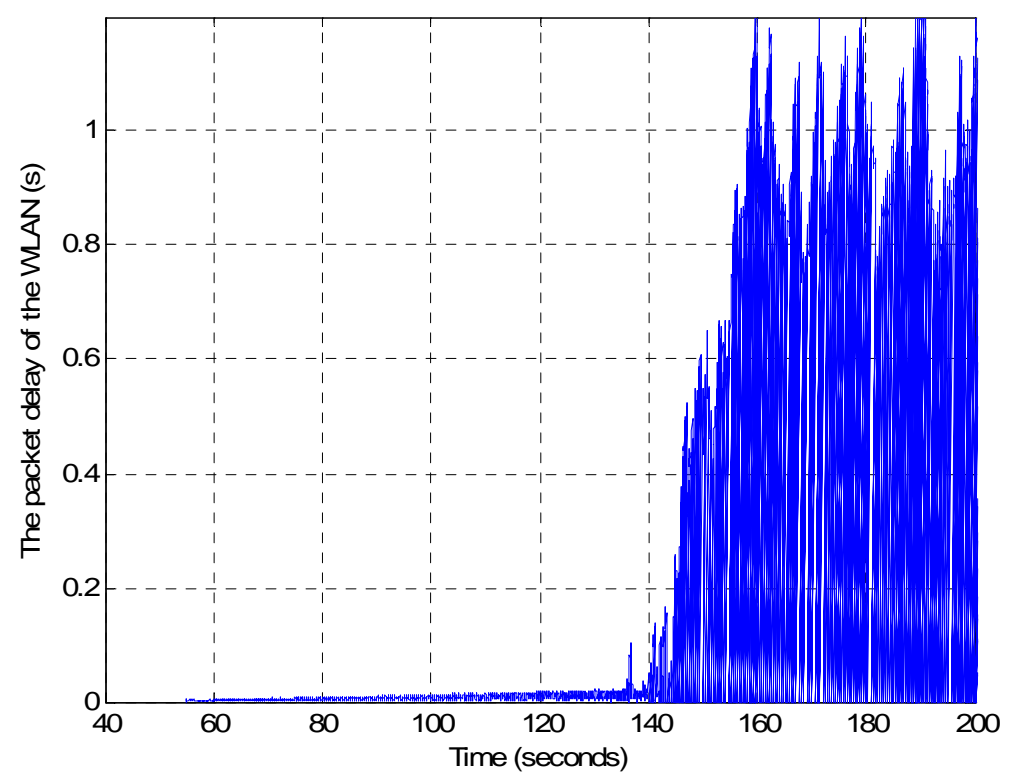

(a) Without vertical handoff.

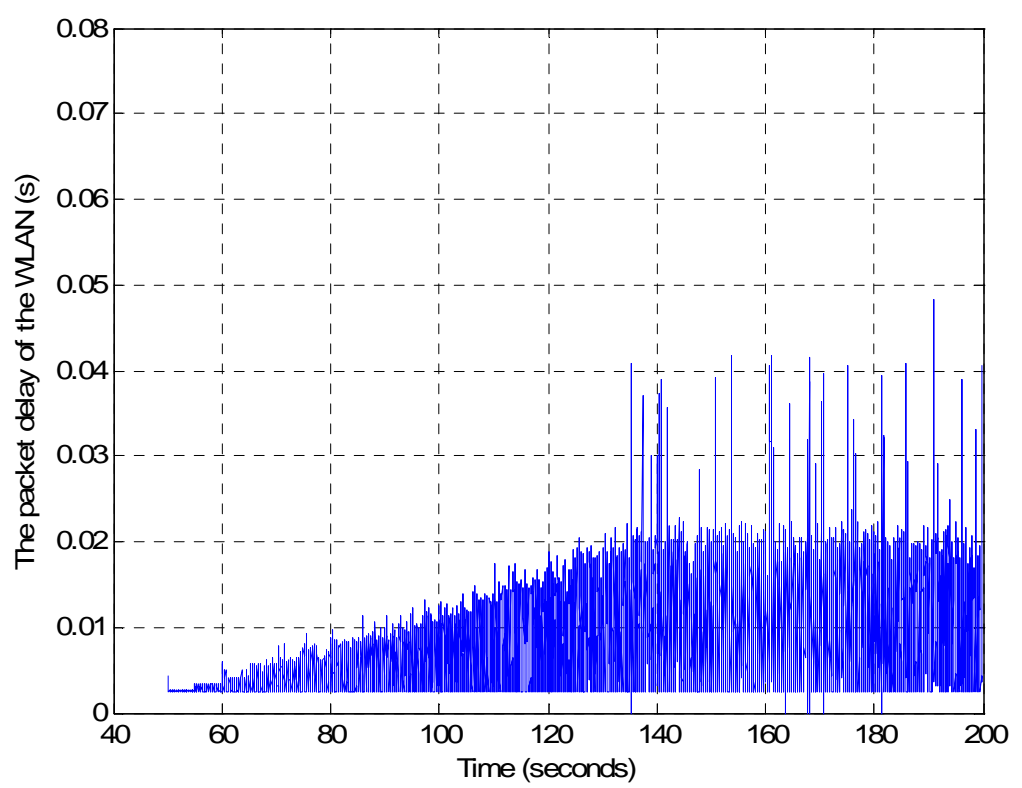

(b) With vertical handoff.

Figure 5.4: The packet delay of the first MN during the vertical handoff jointly with admission control.

contention based MAC mechanism in the WLAN. It is also observed that the packet delay in the WLAN is larger than the one in the WiMAX. This is an ideal case since we set maximum traffic load with QoS support in the WLAN and there is no 
traffic load in the WiMAX network before the MN is handed off to WiMAX in the simulation. If more MNs switch to WiMAX, the gap of the packet delay between WLAN and WiMAX will be decreased. In the simulation, if a packet is lost, we set the packet delay for the packet to zero. Although there are two packets lost, which is observed in Figure 5.6, it occurs not due to the handoff but the saturate status of the WLAN. When the MN moves back and the RSS is larger than the threshold, the MN switches to the WLAN and the packet delay resumes. During the movement of the MN, the system throughput is not affected by the handoff as shown in Figure 5.7.

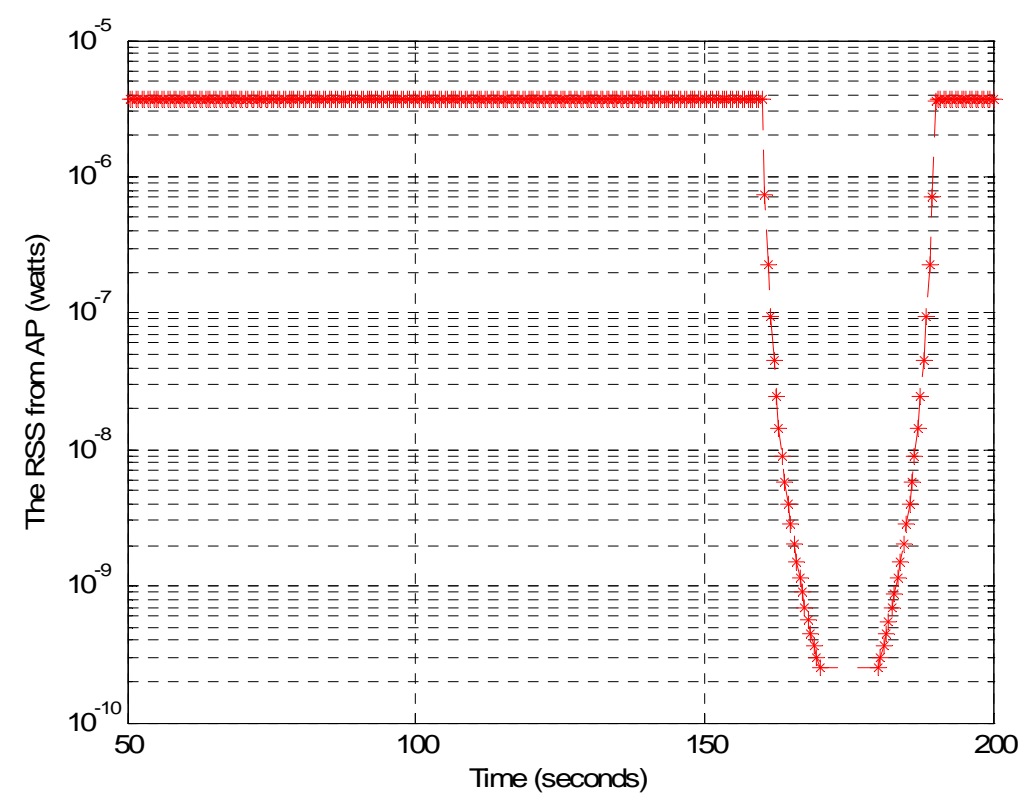

Figure 5.5: The RSS from AP When the first MN is moving out/in the doublecoverage area.

\section{Handoff delay and signaling cost}

In general, handoff delay consists of L2 handoff delay and L3 handoff delay. The L2 handoff delay incurs during the new network scanning and entry procedure. The L3 handoff delay results from the stage of new access router discovery and route information update. In traditional horizontal handoff, the MN only has one 


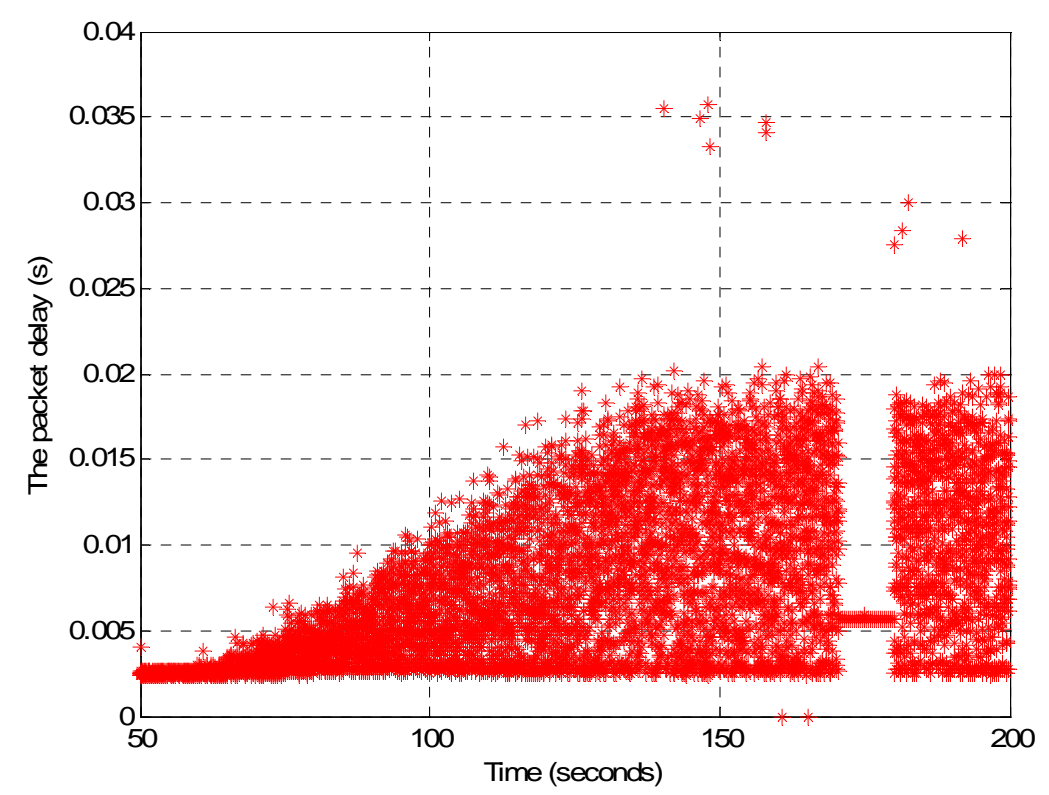

Figure 5.6: The packet delay of the first MN When it is moving out/in the doublecoverage area.

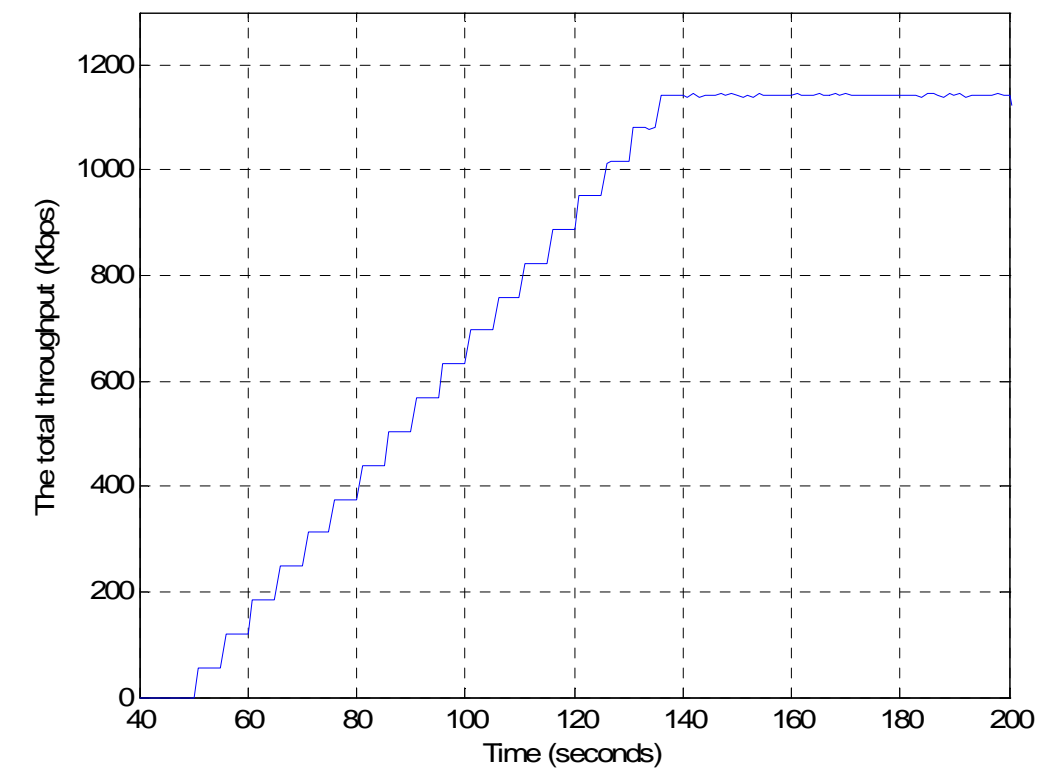

Figure 5.7: The system throughput When the first MN is moving out/in the doublecoverage area.

network interface and the L2 delay is always a part of the handoff delay. Sometimes it takes a long time to finish L2 handoff. For example, when an MN scans for a 
new 802.16 BS, it needs to receive the DL-MAP, DCD, UCD and UL-MAP MAC management messages. The DCD and UCD MAC messages are transmitted by the BS periodically. The maximum interval is $10 \mathrm{~s}$ in the standard [26]. In our simulation, with the 802.16 model from NIST [43], the DCD and UCD interval is set to $5 \mathrm{~s}$. However in vertical handoff, the MN has two independent radio interfaces which can operate simultaneously. As a result, the L2 handoff which is to set up the new link in the MAC level can complete before the handoff decision. Hence, we define the handoff delay as the time period from the instant to start a handoff decision making to the instant to send or receive information packets via the new link. That is the handoff delay is mainly from L3 handoff. There are many factors which can affect the L3 handoff delay, such as the link delay in the backbone and the topology of the WMRs.

Table 5.2: Signaling cost and delay in Layer 3 handoff

\begin{tabular}{|c|c|c|}
\hline Scenario & Layer 3 Handoff Signaling Cost & Layer 3 Handoff Delay \\
\hline Case 1 & (RtSolPr, PrRtAdv) 1, (URI, URI-ACK) 1 & $2 T_{802.11}+2 T_{802.16}$ \\
\hline Case 2 & (RtSolPr, PrRtAdv) 1, (URI, URI-ACK) 3 & $2 T_{802.11}+2 T_{802.16}+4 T_{l i n k}$ \\
\hline Case 3 & (RtSolPr, PrRtAdv) 1, (FBU, FBack) 1, & $2 T_{802.11}+2 T_{M N-H A}+2 T_{H A-F A}$ \\
& (HI, HAck) 1,(URI, URI-ACK) n & $+2 T_{H A-W M R 3}+2 T_{M N-F A}$ \\
\hline
\end{tabular}

Also, the signaling cost associated with the L3 handoff depends on the handoff scenarios. Table 5.2 gives the average L3 handoff delay and signaling cost for the different scenarios. In the table, $T_{802.11}$ is the one-way average packet delay on the 802.11 link. Its value depends on the system traffic load due to the contention mechanism of 802.11 channel access. In [44], the average packet delay in the WLAN is bounded from $5 \mathrm{~ms}$ to $30 \mathrm{~ms}$ according to different collision probabilities when the MN is transmitting a packet. In the simulation, when the WLAN has the maximum number of the MNs who have voice traffic to transmit with QoS support, $T_{802.11}$ is measured to be $8.3 \mathrm{~ms}$. It is a reasonable value as compared with analytical results in [44]. The frame interval in 802.16 is set to be $4 \mathrm{~ms}$. $T_{802.16}$ is the oneway average packet delay on the 802.16 link, which is measured to be $3.669 \mathrm{~ms}$ in the simulation. The variable $T_{\text {link }}$ is the one-way link delay between the wired 
nodes, which is set to be $1 \mathrm{~ms}$. The value of $n$ for calculating the handoff delay depends on the topology of the wireless mesh backbone. In Case 1, $n$ is equal to zero since there are no URI messages in the backbone. The average L3 handoff delay is calculated to be $23.938 \mathrm{~ms}$. In Case 2, the URI is sent from WMR5 to WMR9 and from WMR9 to WMR4. Therefore $n$ is equal to four. The average L3 handoff delay is $27.938 \mathrm{~ms}$ in Case 2. The RtSolPr-PrRtAdv and URI-URIACK exchanges in the wireless link are implemented in the simulation. The L3 handoff delay depends on not only the wireless link delay, but also the backbone topology. The vertical handoff delay in Case 1 is lower than that in Case 2 as expected, because there is no routing information exchange between the WMRs. The edge WMR in cases 1 and 2 is equivalent to the MAP in HMIPv6 [10]. The differences are that the MNs in HMIPv6 need to configure two two CoAs. In the proposed handoff scheme, the MN uses the same IP address in the micro-mobility scenario and there is no CoA assignment. Therefore the IP address acquirement and configure time is saved. In addition, when the MN performs handoff between the Intra Mesh Routers, i.e. in case 2, it is not necessary to inform the HA about the updating route information. The updating route information only goes to the neighbors of the Intra Mesh Routers. Obviously, the delay while the MN sends updating route information to the $\mathrm{HA}$ is larger than that when the $\mathrm{MN}$ sends updating route information to neighbor mesh routers of the attach point. This depends on the topology of the network. In general, it still needs several hops to reach the HA. Hence, from this point, the delay of updating route information is reduced. As a result, the proposed vertical handoff scheme can provide a lower handoff delay in the micro-mobility scenario.

Although there are some L3 handoff delay investigations in the literature, since each of them is based on different assumptions on the environment, topology, link delays and even definition of the handoff delay, it is not possible to compare the results directly. Case 3 indicates the scenario of handoff between Inter Mesh Routers as shown in Figure 4.4. It is a macro-mobility scenario. The FBU, FBack, HI and HAck are binding update messages in FMIPv6 [28]. In this case, the handoff suffers 
from message transmitting time for updating the route from the MN to the HA $\left(T_{M N-H A}\right)$ and from the HA to the FA $\left(T_{H A-F A}\right)$. The packet delay from MN to HA $\left(T_{M N-H A}\right)$ includes one $T_{802.11}$ and three $T_{\text {link }}$ (assuming that the link delay between HA and WGW2 is the same as the one between MGW2 and WMR8). We set the link delay between HA and FA to be $5 \mathrm{~ms}$ [45]. The total handoff delay yields

$$
\begin{aligned}
& 2 T_{802.11}+2 T_{M N-H A}+2 T_{H A-F A}+2 T_{H A-W M R 3}+2 T_{M N-F A} \\
& =2 T_{802.11}+2\left(T_{802.11}+3 T_{\text {link }}\right)+2 T_{H A-F A}+2\left(3 T_{\text {link }}\right)+2\left(T_{802.16}+3 T_{\text {link }}\right) \\
& =4 T_{802.11}+2 T_{802.16}+2 T_{H A-F A}+18 T_{\text {link }} .
\end{aligned}
$$

The total handoff delay is calculated to be $68.538 \mathrm{~ms}$. For the worst case in which the MN needs to send DAC message, the extra delay $2 T_{M N-F A}$ should be added to the total handoff delay, which is increased to $81.876 \mathrm{~ms}$. Obviously the handoff delay in the macro-mobility scenario is larger than the one in the micro-mobility scenario. The International Telecommunication Union (ITU) has recommended one way end-to-end packet transmission delay no greater than $150 \mathrm{~ms}$ for good voice call quality, with a limit of $400 \mathrm{~ms}$ for acceptable voice calls. Therefore with proper buffer size control, the voice call can still be well served during the vertical handoff. 


\section{Chapter 6}

\section{Conclusions and Future Work}

\subsection{Conclusions}

In this thesis, vertical handoff between 802.11 and 802.16 wireless access networks is investigated. We mainly focus on the vertical handoff procedure in wireless mesh backbone and the handoff decision to achieve the goal,which is to provide QoS support to as many as possible users.

After presenting an interworking architecture of wireless mesh backbone, we have proposed an effective vertical handoff scheme between 802.11 and 802.16. The vertical handoff occurs in three scenarios: (1) within the same WMR, (2) between Intra Mesh Routers, and (3) between Inter Mesh Routers. The handoff signaling procedure in these different scenarios has been discussed, which reduces the signaling overhead on the backbone and provides a low handoff delay. The handoff decision algorithm combined with admission control can guarantee QoS support to the existing traffic flows in WLAN by transferring new calls to the other network whenever necessary, so as to provide QoS support to as many users as possible. Simulation results demonstrate that the newly proposed vertical handoff scheme performs well with respect to signaling cost, handoff delay, system throughput, and packet delay. 


\subsection{Future Work}

There are many issues that should be further investigated in designing the vertical handoff scheme for the heterogeneous networks.

In this thesis, we only consider the infrastructure mode between the MN and the AP or BS. The MN can only communicate with the AP or BS. However if the network operates in an ad hoc mode, the MN can set up direct connections with its neighbors. Therefore to design a distributed handoff scheme becomes a main challenge.

The power consumption problem for an MN is always a crucial factor. In this thesis the dual-interface of the MN is always on and can operate at the same time at the MAC level. This will waste energy when the MN is using one interface to transmit traffic and the other interface is still in a power on status. But if we power off the other interface, it will lead to a large handoff delay when the handoff is necessary. Therefore how to find a tradeoff between the power consumption and good handoff performance should be studied in the future. 


\section{References}

[1] I. F. Akyildiz, X. Wang, and W. Wang, "Wireless mesh networks: a survey," Computer Networks, vol. 47, no. 4, pp. 445-487, 2005.

[2] M. Buddhikot, G. Chandranmenon, S. Han, Y. Lee, S. Miller, and L. Salgarelli, "Integration of 802.11 and third-generation wireless data networks," in Proc. IEEE INFOCOM 2003, vol. 1, Apr. 2003, pp. 503-512.

[3] J. Song, S. Lee, and D. Cho, "Hybrid coupling scheme for UMTS and wireless LAN interworking," in Proc. IEEE Vehicular Technology Conference, (VTC 2003), vol. 4, Oct. 2003, pp. 2247-2251.

[4] S. Frattasi, E. Cianca, and R. Prasad, "An Integrated AP for Seamless Interworking of Existing WMAN and WLAN Standards," Wireless Personal Communications, vol. 36, no. 4, pp. 445-459, Mar. 2006.

[5] I. Akyildiz, J. McNair, J. Ho, H. Uzunalioglu, and W. Wang, "Mobility management in next-generation wireless systems," Proceedings of the IEEE, vol. 87, no. 8, pp. 1347-1384, Aug. 1999.

[6] C. Perkins, "IP Mobility Support for IPv4," RFC 3344, IETF, Aug. 2002.

[7] D. Johnson, C. Perkins, and J. Arkko, "Mobility Support in IPv6," RFC 3775, IETF, Jun. 2004.

[8] D. Saha, A. Mukherjee, I. Misra, M. Chakraborty, and N. Subhash, "Mobility support in IP: a survey of related protocols," IEEE Network, vol. 18, no. 6, pp. 34-40, Nov./Dec. 2004. 
[9] A. Campbell, J. Gomez, S. Kim, C.-Y. Wan, Z. Turanyi, and A. Valko, "Comparison of IP micromobility protocols," IEEE Wireless Communications, vol. 9, no. 1, pp. 72-82, Feb. 2002.

[10] H. Soliman, C. Castelluccia, K. El-Malki, and L. Bellier, "Hierarchical Mobile IPv6 Mobility Management (HMIPv6)," RFC 4140, IETF, Aug. 2005.

[11] W. Wu, N. Banerjee, K. Basu, and S. K. Das, "SIP-based vertical handoff between WWANs and WLANs," IEEE Wireless Communications, vol. 12, no. 3, pp. 66-72, Jun. 2005.

[12] R. Good and N. Ventura, "A multilayered hybrid architecture to support vertical handover between IEEE 802.11 and UMTS," in Proc. International conference on Wireless Communications and Mobile Computing (IWCMC 2006), 2006, pp. 257-262.

[13] M. Stemm and R. H. Katz, "Vertical handoffs in wireless overlay networks," Mobile Networks and Applications, vol. 3, no. 4, pp. 335-350, 1998.

[14] C. Lee, L. Chen, M. Chen, and Y. Sun, "A framework of handoffs in wireless overlay networks based on mobile IPv6," IEEE Journal on Selected Areas in Communications, vol. 23, no. 11, pp. 2118-2128, Nov. 2005.

[15] A. Hasswa, N. Nasser, and H. Hossanein, "Generic vertical handoff decision function for heterogeneous wireless," in Proc. Second IFIP International Conference on Wireless and Optical Communications Networks (WOCN 2005), Mar. 2005, pp. 239-243.

[16] Y. N. Gyekye and J. I. Agbinya, "Vertical Handoff between WWAN and WLAN," in Proc. International Conference on Networking, International Conference on Systems and International Conference on Mobile Communications and Learning Technologies (ICN/ICONS/MCL 2006), Apr. 2006, pp. 132-137. 
[17] J. Nie, J. Wen, Q. Dong, and Z. Zhou, "A seamless handoff in IEEE 802.16a and IEEE 802.11n hybrid networks," in Proc. International Conference on Communications, Circuits and Systems, vol. 1, May 2005, pp. 383-387.

[18] S. Sharma, I. Baek, Y. Dodia, and T. Chiueh, "Omnicon:a mobile ip-based vertical handoff system for wireless LAN and GPRS links," in Proc. International Conference on Parallel Processing Workshops, Aug. 2004, pp. 330-337.

[19] K. Kim, C. Kim, and T. Kim, "A Seamless Handover Mechanism for IEEE 802.16e Broadband Wireless Access," in Proc. Computational Science (ICCS 2005), vol. 3515, May 2005, pp. 527-534.

[20] H. J. Jang, J. Jee, Y. H. Han, S. D. Park, and J. Cha, "Mobile IPv6 Fast Handovers over IEEE 802.16e Networks," IETF Internet Draft,Work in progress, Aug. 2007.

[21] IEEE 802.21 Working Group. [Online]. Available: http://www.ieee802.org/21/

[22] T. Ali-Yahiya, K. Sethom, and G. Pujolle, "A Case Study: IEEE 802.21 Framework Design for Service Continuity across WLAN and WMAN," in Proc. IFIP International Conference on Wireless and Optical Communications Networks (WOCN 2007), Jul. 2007, pp. 1-5.

[23] S. Mangold, S. Choi, P. May, O. Klein, G. Hiertz, and L. Stibor, "IEEE 802.11e Wireless LAN for Quality of Service," in Proc. European Wireless (EW2002), Florence, Italy, Feb. 2002.

[24] Wireless LAN Medium Access Control (MAC) and Physical Layer (PHY) specifications: Amendment 8: Medium Access Control (MAC) Quality of Service Enhancements, IEEE 802.11e Std., Nov. 2005.

[25] Q. Ni, L. Romdhani, and T. Turletti, "A survey of QoS enhancements for IEEE 802.11 wireless LAN: Research Articles," Wireless Communications and Mobile Computing, vol. 4, no. 5, pp. 547-566, Aug. 2004. 
[26] IEEE Standard for Local and metropolitan area networks, Part 16: Air Interface for Fixed Broadband Wireless Access Systems, IEEE 802.16 Std., 2004.

[27] C. Cicconetti, A. Erta, L. Lenzini, and E. Mingozzi, "Performance Evaluation of the IEEE 802.16 MAC for QoS Support," IEEE Transactions on Mobile Computing, vol. 6, no. 1, pp. 26-38, Jan. 2007.

[28] R. Koodli, "Fast Handovers for Mobile IPv6," RFC 4068, IETF, Jul. 2005.

[29] M. Liebsch, "Candidate Access Router Discovery (CARD)," RFC 4066, IETF, Jul. 2005.

[30] R. Rouil and N. Golmie, "Effects of IEEE 802.16 link parameters and handover performance for select scenarios," IEEE 802.21 Media Independent Handover Working Group Contribution, Mar. 2006.

[31] L. Wang, A. Chen, and H. Chen, "Network Selection with Joint Vertical and Horizontal Handoff in Heterogeneous WLAN and Mobile WiMax Systems," in Proc. IEEE Vehicular Technology Conference (VTC 2007), Apr. 2007, pp. 794-798.

[32] L. Cai, X. Shen, J. Mark, L. Cai, and Y. Xiao, "Voice capacity analysis of WLAN with unbalanced traffic," IEEE Transactions on Vehicular Technology, vol. 55, no. 3, pp. 752-761, May 2006.

[33] A. Trad, F. Munir, and H. Afifi, "Capacity evaluation of VoIP in IEEE 802.11e WLAN environment," in Proc. IEEE Consumer Communications and Networking Conference (CCNC 2006), vol. 2, Jan. 2006, pp. 828-832.

[34] D. Pong and T. Moors, "Call admission control for IEEE 802.11 contention access mechanism," in Proc. IEEE Global Telecommunications Conference (GLOBECOM 2003), vol. 1, Dec. 2003, pp. 174-178.

[35] Y. Xiao and H. Li, "Voice and Video Transmissions with Global Data Parameter Control for the IEEE 802.11e Enhance Distributed Channel Access," IEEE Trans. Parallel Distrib. Syst., vol. 15, no. 11, pp. 1041-1053, Nov. 2004. 
[36] H. Zhai, X. Chen, and Y. Fang, "A call admission and rate control scheme for multimedia support over IEEE 802.11 wireless LANs," Wireless Networks, vol. 12, no. 4, pp. 451-463, Jul. 2006.

[37] N. Hegde, A. Proutiere, and J. Roberts, "Evaluating the voice capacity of 802.11 WLAN under distributed control," in Proc. The 14th IEEE Workshop on Local and Metropolitan Area Networks (LANMAN 2005), Sep. 2005.

[38] D. Zhao and X. Shen, "Performance of packet voice transmission using IEEE 802.16 protocol," IEEE Wireless Communications, vol. 14, no. 1, pp. 44-51, Feb. 2007.

[39] K. Wongthavarawat and A. Ganz, "Packet scheduling for QoS support in IEEE 802.16 broadband wireless access systems," International Journal of Communication Systems, vol. 16, no. 2, pp. 81-96, Feb. 2003.

[40] O. Yang and J. Lu, "Call Admission Control and Scheduling Schemes with QoS Support for Real-time Video Applications in IEEE 802.16 Networks," Journal of Multimedia (JMM), vol. 1, pp. 21-29, May 2006.

[41] Network Simulator NS-2 (2.31), UCB/LBNL/VINT. [Online]. Available: http://www.isi.edu/nsnam/ns/

[42] IEEE 802.11e EDCA Simulation Model for NS-2. [Online]. Available: http://www.tkn.tu-berlin.de/research/802.11e_ns2/

[43] IEEE 802.16 Simulation Model for NS-2, NIST. [Online]. Available: http://www.antd.nist.gov/seamlessandsecure.shtml

[44] H. Zhai, X. Chen, and Y. Fang, "How well can the IEEE 802.11 wireless LAN support quality of service," IEEE Transactions on Wireless Communications, vol. 4, no. 6, pp. 3084-3094, Nov. 2005.

[45] T. Kwon, M. Gerla, and S. Das, "Mobility management for VoIP service: Mobile IP vs. SIP," IEEE Wireless Communications, vol. 9, no. 5, pp. 66-75, Oct. 2002. 